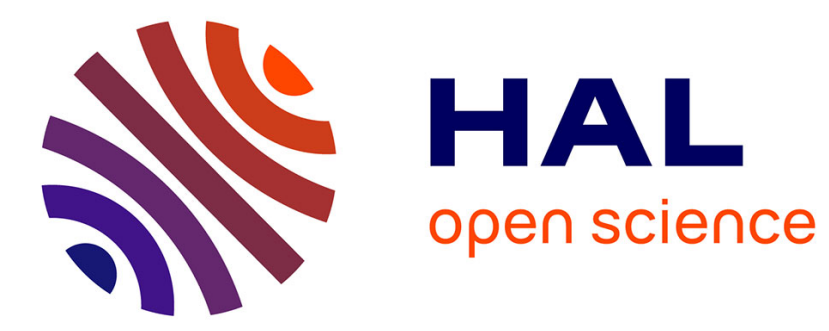

\title{
Louis Pasteur ou l'entreprise scientifique au temps du capitalisme industriel
}

Gabriel Galvez-Behar

\section{To cite this version:}

Gabriel Galvez-Behar. Louis Pasteur ou l'entreprise scientifique au temps du capitalisme industriel. Annales. Histoire, Sciences sociales, 2018, 73 (3), pp.629-656. 10.1017/ahss.2019.46 . halshs$01267638 \mathrm{v} 5$

\section{HAL Id: halshs-01267638 \\ https://shs.hal.science/halshs-01267638v5}

Submitted on 29 Oct 2019

HAL is a multi-disciplinary open access archive for the deposit and dissemination of scientific research documents, whether they are published or not. The documents may come from teaching and research institutions in France or abroad, or from public or private research centers.
L'archive ouverte pluridisciplinaire HAL, est destinée au dépôt et à la diffusion de documents scientifiques de niveau recherche, publiés ou non, émanant des établissements d'enseignement et de recherche français ou étrangers, des laboratoires publics ou privés. 


\title{
LOUIS PASTEUR OU L'ENTREPRISE SCIENTIFIQUE AU TEMPS DU CAPITALISME INDUSTRIEL
}

\author{
GABRIEL GALVEZ-BEHAR
}

Manuscrit d'auteur au 30 avril 2016 - Pre-print version 04/30/2016

La version définitive - et fortement remaniée - de ce manuscrit a été publié sous le titre "Louis Pasteur ou l'entreprise scientifique au temps du capitalisme industriel » in Annales. Histoire, Sciences sociales, 2018, 73 (3), pp.629-656 ; https://doi.org/10.1017/ahss.2019.46

The final - and significantly revised - version of this manuscript was published under the title "Louis Pasteur ou l'entreprise scientifique au temps du capitalisme industriel $\gg$ in Annales. Histoire, Sciences sociales, 2018, 73 (3), pp.629-656. https://doi.org/10.1017/ahss.2019.46

Le prétendu tournant néo-libéral qui a frappé les institutions scientifiques depuis les années

1980 a suscité de nombreux travaux, attachés à montrer combien la frontière entre science et économie était devenue poreuse ${ }^{1}$. A contrario d'autres analyses, maintenant nourries par plusieurs décennies de science studies, nous ont enseigné que la science n'a jamais été pure².

Paradoxalement, une histoire économique des mondes savants qui s'attacherait à étudier, dans

la longue durée, la dimension économique de l'activité scientifique reste encore rare. Une telle

histoire a de multiples aspects, de l'étude des rapports entre sciences et industries à celle du

1. Sheila Slaughter et Larry L Leslie, Academic capitalism : politics, policies, and the entrepreneurial university, Baltimore, Johns Hopkins University Press, 1997 ; Rebecca Lave, Philip Mirowski et Samual Randalls, «Introduction: STS and Neoliberal Science», Social Studies of Science, vol. 40, n 5, 2010, p. 659-675 ; Philip Mirowski, Science-mart : privatizing American science, Cambridge, Mass., Harvard University Press, 2011 ; Elizabeth Popp Berman, Creating the market university how academic science became an economic engine, Princeton, Princeton University Press, 2012. Sur le tournant néo-libéral, Brigitte Gaïti, «L'érosion discrète de l'État-providence dans la France des années 1960 », Actes de la recherche en sciences sociales, $\mathrm{n}^{\circ}$ 201-202, 2014, p. 58-71.

2. Steven Shapin, The Scientific Life : A Moral History of a Late Modern Vocation, Chicago, University of Chicago Press, 2008 ; ID., Never Pure, Baltimore, Johns Hopkins University Press, 2010.

(C) Gabriel Galvez-Behar Version de pré-publication/Pre-print version 
financement des activités savantes. Elle prend ici la forme d'une contribution à l'étude de l'entreprise scientifique de Louis Pasteur ${ }^{3}$.

Dire que Pasteur fut un entrepreneur n'est pas chose nouvelle. Les nombreux travaux qui se rapportent à ce héros de la science conquérante ont maintes fois souligné son caractère entreprenant. Entreprenant vis-à-vis des objets : tartre, ferments, moutons, microbes. Entreprenant à l'égard des hommes : collaborateurs, détracteurs ou protecteurs. Entreprenant vis-à-vis des institutions : facultés, sociétés savantes ou Institut éponyme. Homme de science entreprenant, Louis Pasteur fut aussi un savant industrieux, côtoyant brasseurs et sériciculteurs, industriels et banquiers. Là encore, les travaux ne manquent pas qui le campent en garant d'une sainte alliance entre la science et l'industrie où la première n'hésite pas à emprunter les voies tracées par la seconde pourvu que celle-ci obéisse finalement à celle-là .

Il ne suffit cependant pas d'être entreprenant et industrieux pour recevoir la qualification d'entrepreneur. Cette notion est d'un maniement difficile du fait de la multiplicité de ses usages et de ses justifications théoriques. Des entrepreneurs de morale aux entrepreneurs de réputation en passant par les academic entrepreneurs, le concept menace de perdre en

3. Les travaux sur Pasteur sont pléthoriques. Nous avons privilégié : Claire Salomon-Bayet, Pasteur et la révolution pastorienne, Paris, Payot, 1986 ; Bernardino Fantini et Michel Morange (dir.), L'Institut Pasteur : contributions à son histoire, Paris, Ed. La Découverte, 1991 ; Pierre Darmon, Pasteur, Paris, Fayard, 1995 ; Maurice Vallery-Radot, Pasteur, Paris, Perrin, 1994 ; François Dagognet, Pasteur sans la légende, Le PlessisRobinson, Synthélabo, 1994 ; Patrice Debré, Louis Pasteur, Paris, Flammarion, 1997 ; Gerald L. Geison, The Private Science of Louis Pasteur, Princeton, N.J., Princeton University Press, 1995 ; Bruno Latour, Pasteur : guerre et paix des microbes, Paris, La Découverte, [1984] 2011.

4. Guillaume Carnino, «Louis Pasteur. La science pure au service de l'industrie », Le Mouvement Social, $\mathrm{n}^{\circ} 248,2014$, p. 9-26 ; ID., L'invention de la science. La nouvelle religion de l'âge industriel, Paris, Le Seuil, 2015.

(C) Gabriel Galvez-Behar 
compréhension ce qu'il gagne en extension ${ }^{5}$. De récents travaux offrent cependant un cadre analytique suffisamment robuste pour discriminer des usages plus ou moins pertinents. Ils proposent de déplacer le regard de la figure individuelle de l'entrepreneur vers l'activité entrepreneuriale qui associe une logique entrepreneuriale - dont la projection vers l'avenir, l'incertitude, la recherche de contrôle et la dimension narrative sont les traits essentiels - et un contexte spécifique, constitué de ressources ou d'appuis ${ }^{6}$.

Immanquablement, la figure de Pasteur évoque celle des «chercheurs entrepreneurs » actuels $^{7}$. Porteur de plusieurs projets - combattre, par la science, les maladies de la vigne, de la soie, des hommes -, Pasteur affronta des risques aussi bien pour sa carrière que pour sa vie et celle des autres. Il assura une forme de contrôle sur le monde - le monde des microbes, des vers à soie, des moutons mais aussi des malades - sans oublier de (faire) mettre en récits toute son action. Mobilisant les ressources de plusieurs mondes - le milieu savant, le monde politique, les milieux d'affaires -, Louis Pasteur fut incontestablement un entrepreneur.

Toutefois, au-delà de la caractérisation de Pasteur comme un entrepreneur, il reste à reconstituer la singularité de sa logique entrepreneuriale en essayant de comprendre comment

5. Mark Casson, Bernard Yeung, Anuradha Basu et Nigel Wadeson (dir.), The Oxford Handbook of Entrepreneurship, Oxford, Oxford University Press, 2009 ; Marcelo Bucheli et Rohit Daniel Wadhwani (dir.), Organizations in time : history, theory, methods, Oxford, Oxford University Press, 2014. Sur l'entrepreneuriat scientifique : Henry Etzkowitz, « Entrepreneurial scientists and entrepreneurial universities in American academic science », Minerva, vol. 21, $\mathrm{n}^{\circ} 2-3,1983$, p. 198-233 ; W. Bernard Carlson, «Academic Entrepreneurship and Engineering Education : Dugald C. Jackson and the MIT-GE Cooperative Engineering Course, 1907-1932 », Technology and Culture, 1988, vol. 29, n 3, p. 536-567.

6. Pierre-Marie Chauvin, Michel Grossetti et Pierre-Paul Zalio (dir.), Dictionnaire sociologique de l'entrepreneuriat, Paris, Presses de Sciences Po, 2014, p. 22.

7. Jean-Paul Gaudillière, «Une manière industrielle de savoir » in Dominique Pestre (dir.), Histoires des sciences et des savoirs, vol. 3, Christophe Bonneuil et Dominique Pestre (dir.), Le siècle des technosciences, Paris, Le Seuil, 2015, p. 102. 
elle articule des dimensions différentes et, notamment, une dimension économique. Bien entendu, cela renvoie aux valeurs de la science pasteurienne et aux formes de son désintéressement. Cela conduit surtout à se concentrer sur les ressources qui possèdent une dimension économique évidente ou, du moins, plus évidente que d'autres. N'étudier l'entreprise pasteurienne qu'à l'aune des microbes ou des publications scientifiques, c'est, en effet, prendre le risque d'en construire une représentation tronquée. Aussi trois ressources trop souvent négligées par les études antérieures sont ici au cœur de notre analyse : l'argent, les brevets d'invention et le capital.

Une telle approche se heurte cependant à un problème de sources qu' Ilana Löwy soulignait déjà à propos des travaux de Bruno Latour ${ }^{8}$. Les sources de l'histoire pasteurienne sont à la fois nombreuses et complexes. Nombreuses parce que l'on compte, entre autres, les publications de et sur Pasteur publiées de son vivant mais aussi ses CEuvres et sa correspondance publiées plus tard par sa famille. À ce corpus s'ajoutent les papiers de Pasteur déposés par la famille à la Bibliothèque nationale de France puis les archives conservées dans différentes institutions : l'Académie des sciences, le Musée Pasteur, l'Institut éponyme. Or chacun de ces ensembles relève de logiques de construction précises. Ainsi, comme l'a déjà fait remarquer Guillaume Carnino, la correspondance publiée de Pasteur est expurgée d'un certain nombre de documents voire, au sein de certaines lettres, de certains passages sans que

8. Ilana Löwy, « On hybridizations, networks and new disciplines : The Pasteur Institute and the development of microbiology in France », Studies in History and Philosophy of Science Part A, vol. 25, n 5, 1994, p. 655-688. 
ces coupes ne soient réellement justifiées ${ }^{9}$. Les papiers déposés à la Bibliothèque nationale font apparaître des absences. Tout porte à croire que les sources publiées ou déposées dans ces institutions l'ont été de façon à ne pas porter atteinte au grand récit pasteurien. Pour aller audelà de la légende, l'historien doit refaire son métier.

Aussi est-ce non seulement sur la relecture de sources connues mais encore sur la base de sources nouvelles que repose cette contribution à une histoire économique de l'entreprise pasteurienne. Elle se déroule ici en trois temps. Comprendre quel fut le statut de l'argent dans cette entreprise doit non seulement permettre de définir son rapport au désintéressement mais aussi de mesurer la place occupée par des considérations matérielles dans le développement de la science pasteurienne. Se pencher sur les brevets d'invention doit ensuite éclairer les formes d'appropriation de cette dernière mais aussi son exploitation à des fins commerciales. Enfin établir les liens de cette entreprise scientifique avec le capital doit permettre d'en mesurer la dimension proprement économique.

\section{LE NERF DE LA GUERRE}

L'argent est un des points d'appui de l'entreprise scientifique qu'une tendance relativement récente de l'historiographie invite à suivre à la trace ${ }^{10}$. Une telle approche contraste toutefois avec une longue tradition incarnée par Robert $K$. Merton et qui considérait le désintéressement comme l'une des normes essentielles de l'institution scientifique ${ }^{11}$. Encore

9. Guillaume Carnino, « Louis Pasteur. La science pure au service de l'industrie », Le Mouvement Social, art. cit.

10. David Edgerton, « Time, Money, and History », Isis, vol. 103, n² 2, 2012, p. 316-327.

11. Robert K. Merton, «The Normative Structure of Science », in R. K. Merton, The Sociology of Science. Theoretical and Empirical Investigations, Chicago, The University of Chicago Press, 1973, p. 267-278.

(C) Gabriel Galvez-Behar

Version de pré-publication/Pre-print version 
faut-il remarquer que, chez Merton, qui lui a donné sa dimension cardinale, cette catégorie du désintéressement n'avait pas nécessairement le sens qu'une traduction rapide lui confère ${ }^{12}$. Elle renvoie à une fonction bien précise qui conduit chaque savant à incorporer la surveillance de ses pairs censés sanctionner les fraudes et les comportements opportunistes, même si, bien entendu, une totale conformité aux attentes collectives est impossible et le fait même d'être engagé dans le champ scientifique rend nécessaire une forme d'intéressement. Depuis longtemps d'ailleurs, les normes et les valeurs mertoniennes de la science ne sont plus interprétées que comme des pôles d'un faisceau de tensions qui traversent l'activité scientifique $^{13}$. L'argent participe de cette mise en tension et de cette déstabilisation de la catégorie du désintéressement. Actif non spécifique par excellence, qui peut être échangé contre le plus grand nombre d'objets possible, l'argent peut être réinvesti dans des champs différents. On ne sait donc jamais l'intérêt qu'il peut servir. Aussi la recherche de l'argent suscite-t-elle toujours une incertitude voire un soupçon. Pour cette raison, son usage dans l'entreprise scientifique suppose qu'il soit rendu compatible avec la norme de désintéressement.

\section{Désintéressement et liberté}

Louis Pasteur est indubitablement une figure de la science désintéressée. Le 20 juin 1995, à

l'occasion du centenaire de la mort de Louis Pasteur, son successeur au fauteuil numéro 17 de l'Académie française, Jacques-Yves Cousteau, prononça un vibrant hommage de son

12. En anglais le terme de desinterestedness signifie le détachement vis-à-vis de considérations personnelles mais aussi l'impartialité voire l'indifférence.

13. Ian I. Mitroff, « Norms and Counter-Norms in a Select Group of the Apollo Moon Scientists : A Case Study of the Ambivalence of Scientists », American Sociological Review, vol. 39, n 4, 1974, p. 579-595.

(c) Gabriel Galvez-Behar Version de pré-publication/Pre-print version 
prédécesseur «parfait exemple du don de soi » qui « toute sa vie [avait sacrifié] ses intérêts personnels sur l'autel de la Connaissance, appuyé par sa foi en la science, la France, et la démocratie. ${ }^{14} \mathrm{Ce}$ discours faisait ainsi écho à celui prononcé plus d'un siècle auparavant par Renan qui accueillait Pasteur parmi les Immortels louant une «vie austère, toute consacrée à la recherche, $[\ldots]$ la meilleure réponse à ceux qui regardent notre siècle comme déshérité des grands dons de l'âme. ${ }^{15}$ Le désintéressement fut ainsi une valeur souvent mise en avant pour qualifier l'œuvre pasteurienne puis transmise, au sein de l'Institut Pasteur, aux différentes générations de la famille pasteurienne ${ }^{16}$.

À plusieurs reprises, Pasteur avait eu l'occasion de s'exprimer sur cette question. À l'automne 1865, à la veille de publier ses Études sur le vin, Pasteur avait été invité à séjourner auprès Napoléon III pour lui présenter les conclusions de ses travaux. Au palais impérial, à Compiègne, dans un espace mondain à mille lieues du laboratoire de la rue d'Ulm, il fut en fait question de science et de profit. Dès le début de son séjour, Pasteur avait eu avec l'Impératrice une conversation sur « l'utilité qu'il y aurait eu à ce que les leçons publiques fussent payées comme en Angleterre et au profit des professeurs ${ }^{17}$. Il suggéra qu'il n'y aurait pas en France de professeurs de mérite qui voudraient agir de la sorte, idée que l'Impératrice considéra comme un préjugé. Quelques jours plus tard, le lendemain d'un spectacle de

14. Jacques-Yves Cousteau, Pasteur, le don de soi. Séance solennelle à l'occasion du centenaire de la mort de Louis Pasteur | Académie française, http://www.academie-francaise.fr/pasteur-le-don-de-soi-seancesolennelle-loccasion-du-centenaire-de-la-mort-de-louis-pasteur, consulté le 18 décembre 2015.

15. Louis Pasteur et Ernest Renan, Discours de réception de M. Louis Pasteur. Réponse de M. Ernest Renan, Paris, Calmann-Lévy, 1882, p. 33.

16. Sandra Legout, «La famille pasteurienne en observation : histoire et mémoire », Histoire, économie et société, vol. 20, $\mathrm{n}^{\circ}$ 3, 2001, p. 339-354, p. 340.

17. Lettre de Louis Pasteur à sa femme, 30 novembre 1865, Louis Pasteur, Correspondance de Pasteur, 18401895, Paris, Flammarion, 1951, vol. 2, p. 220.

(c) Gabriel Galvez-Behar Version de pré-publication/Pre-print version 
prestidigitation, Pasteur procédait devant les souverains à des démonstrations après avoir fait venir au palais impérial quelques instruments de son laboratoire. Sur la scène de cour, l'académicien avait su faire le spectacle.

De retour à Paris, Pasteur se sentit obligé de revenir sur cet épisode dans une lettre bien connue écrite à l'aide de camp de l'Empereur, le général Favé ${ }^{18}$. Il entendait justifier la position qu'il avait défendue auprès de l'Impératrice qui « l'avait encouragé à tirer profit de ses études. ${ }^{19}$ Pasteur affirma alors au général Favé que « le savant qui se laisse aller à la tentation d'applications industrielles cesse par là même d'être l'homme de la science pure, complique sa vie et l'ordre habituel de ses pensées de préoccupations qui paralysent en lui tout esprit d'invention pour l'avenir. $»^{20}$ Plus loin, priant son interlocuteur de relayer son message auprès de l'Empereur afin « qu'il ne croie pas que la science désintéressée n'a pour mobile qu'un préjugé », Pasteur conclut qu'il « faut encourager le désintéressement scientifique parce qu'il est une des sources vives du progrès dans la théorie, d'où émane tout progrès dans l'application $»^{21}$. Sans doute est-ce précisément parce que ses recherches avaient une portée pratique - voire économique - évidente que Pasteur ressentit la nécessité de fixer sa doctrine en la matière. En matière de science, le désintéressement était pour Pasteur la garantie de la liberté et de l'inventivité.

18. Lettre de Pasteur au général Favé, 19 décembre 1865, Ibid., p. 236-238. Nous ne savons pas, cependant, où est conservé l'original de cette lettre.

19. Ibid., p. 237.

20. Idem.

21. Ibid., p. 238.

(C) Gabriel Galvez-Behar 


\section{Des besoins du foyer aux nécessités de la science}

La lettre au général Favé porte cependant le témoignage d'une tension qui éclaire le rapport que Pasteur pouvait entretenir à l'argent. Dans un accès de sincérité envers celui qui appuie ses recherches depuis quelques années, Pasteur déclare :

« Il y a des moments pénibles, c'est vrai, ceux où je songe à des dots que mes deux chères petites filles pourront bien ne jamais avoir, ceux où je pense que la mort pourrait leur laisser pour héritage l'aumône de la Société des Amis des Sciences ; mais il y a aussi ceux que je ne peux croire égoïstes à l'égard de mes enfants, de la joie de découvrir et de grandir par des progrès nouveaux, et de servir mon pays sans arrière-pensée d'intérêt personnel. $»^{22}$

Il convient de ne pas négliger l'expression de cette angoisse de Pasteur pour le devenir de sa famille ni, plus généralement, les considérations d'économie domestique. Fils de tanneur, Pasteur avait épousé en 1849 Marie Laurent, fille du recteur de l'académie de Strasbourg où il était alors en poste. S'il n'était pas rare qu'existât un tel écart social, il faut toutefois noter que son épouse apportait alors une dot dix fois supérieure à sa propre contribution ${ }^{23}$. Il lui fallait donc trouver des revenus à la hauteur des espoirs placés dans ce mariage et prendre des dispositions pour sauvegarder les intérêts de sa famille en cas de coup dur. Pour cette raison d'ailleurs, Pasteur souscrivit plusieurs contrats d'assurance-vie ${ }^{24}$. Lorsqu'en octobre 1872, il fut amené à solliciter auprès de Thiers une récompense nationale à l'occasion de son départ en retraite, Pasteur ne manqua d'évoquer les sacrifices qui l'avaient empêché de « recueillir pour [sa] famille et pour [lui-même] le fruit de ses efforts. ${ }^{25}$ Ainsi, malgré son désintéressement

\section{Ibid.}

23. Selon le contrat de mariage rappelé dans la liquidation des communauté et succession de Louis Pasteur, ce dernier apporta 2000 francs à la communauté tandis que Marie Laurent contribuait à hauteur de 20000 francs, 10000 supplémentaires étant prévu à la mort de son père. Archives nationales, Minutier central, Liquidation des communauté et succession de Louis Pasteur, 13 mars 1896, MI/RS/1173, [ET/XLIII/1500].

24. Archives nationales du monde du travail, 47 AQ 56 et 182 AQ 7.

25. L. Pasteur, Correspondance de Pasteur, 1840-1895, op. cit., p. 566.

(C) Gabriel Galvez-Behar Version de pré-publication/Pre-print version 
de savant, Pasteur savait-il exprimer ses besoins pécuniaires en mettant en avant ses nécessités domestiques.

L'intérêt de Pasteur pour l'argent n'eut cependant pas que des motivations domestiques. Toute son entreprise scientifique fut traversée par des recherches récurrentes de ressources financières qui prirent la forme de candidatures à des concours ou celle d'un recours souvent subtil à de multiples patronages. Ces récompenses permettaient à Pasteur de financer ses recherches même si elles ne l'empêchaient pas de solliciter d'autres personnes ${ }^{26}$. En fait, dans la première partie de sa carrière, Pasteur combine trois sources de financement : les prix, les subventions obtenues auprès des ministères et ses ressources personnelles ${ }^{27}$. La publication de ses Études sur le vin (1866) puis de ses Études sur la maladie des vers à soie (1870) illustre bien la dimension économique de cette entreprise scientifique. Dans une lettre adressée à Victor Duruy, Pasteur n'hésita pas à faire la réclame de son ouvrage, déclarant :

"L'impression de l'ouvrage est très soignée. Elle a été faite entièrement à mes frais à l'Imprimerie impériale. La plupart des planches sont en taille douce et en couleur. Je serais heureux que Votre Excellence voulût bien souscrire pour un certain nombre d'exemplaires. Le prix de l'ouvrage est de $15 \mathrm{fr} .{ }^{28}$

Quatre ans plus tard, lorsqu'il publia, à ses frais toujours, ses Études sur la maladie du vers à soie, Pasteur sollicita le ministre de la Maison de l'Empereur, le maréchal Vaillant, afin d'obtenir une souscription sur les fonds de son ministère. À cette occasion, Pasteur n'hésita pas à mettre en lumière les coûts de l'opération :

26. G. L. Geison, The Private Science of Louis Pasteur, op. cit., p. 34.

27. Lettre de Pasteur au ministre d'État, 13 août 1861, L. Pasteur, Correspondance de Pasteur, 1840-1895, op. cit., p. 89.

28. Lettre de Pasteur à Victor Duruy, 22 septembre 1866, Ibid., p. 288.

(C) Gabriel Galvez-Behar $\quad$ Version de pré-publication/Pre-print version 
«Les frais qu'il m'a occasionnés sont considérables ; quand j'aurai réuni toutes les factures, leur total, je le crains, ne sera pas moindre de 35 à 40000 francs. Les gravures, dessins, reproductions photographiques dont je l'ai orné ont singulièrement accru sa dépense. Le sujet, la clarté de l'exposition, une ressemblance désirable avec mes Études sur le vin dédiées à l'Empereur, le voulaient ainsi. Lorsque sur le prix de 20 fr., déjà bien élevé, le libraire aura pris un tiers et l'imprimerie le prix de revient de chaque exemplaire, il restera peu de chose pour l'auteur ; mais nous autres savants pour la gloire et point pour la fortune $»^{29}$

Malgré cette nouvelle déclaration de désintéressement, Pasteur était inquiet du succès de son ouvrage. Il espérait que l'opinion de l'Impératrice, à qui il l'avait fait remettre, fût favorable car les frais s'élevaient à 41000 francs. « Si l'ouvrage ne se vend pas bien, nous perdrons beaucoup » avoua-t-il à son collaborateur Maillot ${ }^{30}$.

En fait, la publication de ces deux ouvrages, qui venaient clore deux cycles de travaux, peut s'analyser comme un double investissement : un investissement financier, d'une part, puisque le coût de l'édition représentait plus de cinq années du traitement fixe d'un professeur de la Faculté des sciences de Paris, mais aussi un investissement épistémique ${ }^{31}$. Ainsi Pasteur fit-il le choix d'apporter d'autant plus d'attention aux planches, aux dessins et aux reproductions de son ouvrage qu'il attachait une place essentielle à l'observation dans son travail. En outre, donner à voir était une manière de susciter la conviction de ses lecteurs. Pasteur ne dit pas autre chose lorsqu'il sollicita en août 1878 une somme de 5000 francs auprès du secrétaire perpétuel de l'Académie des sciences afin de refaire des expériences sur la fermentation, à la suite des doutes suscités par la publication posthume d'un manuscrit de Claude Bernard. Pasteur se disait ainsi « décidé à frapper tous les yeux par les résultats que j'ai l'espoir de

29. Lettre de Pasteur au Maréchal Vaillant, 13 avril 1870, Ibid., p. 476.

30. Lettre de Pasteur à Maillot, 9 mai 1870, Ibid., p. 480.

31. George Weisz, The Emergence of Modern Universities in France, 1863-1914, Princeton, N.J, Princeton University Press, 1983, p. 58. 
soumettre prochainement à l'Académie. $»^{32}$ Le caractère spectaculaire de la science pasteurienne avait un prix.

L'autre élément que met en lumière la publication des deux séries d'Études est l'imbrication de l'économie domestique et de l'économie professionnelle. Ces deux entreprises témoignent, en effet, d'une indistinction entre les ressources familiales et les projets scientifiques. Cette tension et ce souci constant de trouver de l'argent pour protéger les siens - qu'ils soient de sa famille ou de son laboratoire - et pour financer ses recherches contribue à mieux comprendre pourquoi Pasteur prit publiquement position dans les colonnes de la Revue des cours scientifiques pour défendre «le budget de la science ${ }^{33}$. Soutenant l'initiative de Victor Duruy et sa création d'une École pratique des hautes études, Pasteur décrivait le sacrifice des savants souffrant et mourant des maladies contractées dans leurs laboratoires mal chauffés. Il y mettait en lumière la pauvreté des lieux de science mal financés par l'État alors que les laboratoires les mieux dotés s'avéraient appartenir directement aux savants qui les dirigeaient.

Aussi la condition individuelle du savant, dans sa dimension économique et sociale, étaitelle le meilleur point d'appui pour la recherche elle-même. Dès lors, il n'est pas étonnant que Pasteur ait caressé le désir d'être riche comme il en fit l'aveu à son fidèle collaborateur Émile Duclaux :

32. Lettre de Pasteur au secrétaire perpétuel de l'Académie des sciences, 20 août 1878, Louis Pasteur, Correspondance de Pasteur, 1840-1895, Paris, Flammarion, 1951, vol. 3, p. 64.

33. Louis Pasteur, «Le budget de la science », Revue des cours scientifiques de la France et de l'étranger, 1868, vol. $5, \mathrm{n}^{\circ} 9$, p. $137-139$.

(C) Gabriel Galvez-Behar Version de pré-publication/Pre-print version 
«Ah! Que ne suis-je riche, millionnaire. Je vous comblerais des plus beaux appointements et je vous dirais à vous, à Raulin, à Gernez, à Van Tieghem, etc. : Venez, nous allons transformer le monde par nos découvertes. $»^{34}$

Ce généreux esprit de lucre, que ces lignes mettent en relief, ne doit pas être tant considéré comme un trait psychologique que comme une disposition économique visant à accroître ses gains et pour mieux les réinvestir. En effet, au fur et à mesure que s'étire le front des activités de l'entreprise pasteurienne, le besoin d'argent se fait toujours plus sentir.

\section{Récompenser le savant}

Les tractations entreprises par Pasteur pour obtenir des récompenses nationales en 1874 et en 1883 sont aussi le signe de cette quête d'argent qu'il faut toutefois replacer dans un double contexte. Le premier relève d'une histoire longue des récompenses scientifiques qui renvoie aussi bien aux attributions de pensions sous l'Ancien Régime qu'aux prix académiques qu'aux lois de 1791 sur les découvertes. La loi du 7 janvier 1791, qui institue ce que l'on appela un peu plus tard les brevets d'invention, prévoyait également que des récompenses pourraient être dispensées aux inventeurs qui renonceraient à prendre un brevet. Sous la Révolution, une tension se manifesta entre ces deux modes de reconnaissance ${ }^{35}$. Tout au long du XIX ${ }^{\mathrm{e}}$ siècle, le système des prix visant à récompenser le mérite scientifique ou industriel par des corps intermédiaires de l'invention cohabitait avec un système reposant sur le secret ou le brevet et laissant faire le marché. Le recours à la récompense nationale, en revanche, était exceptionnel.

34. Lettre de Pasteur à Duclaux, 29 mars 1871, L. Pasteur, Correspondance de Pasteur, 1840-1895, op. cit., p. 527.

35. Christiane Demeulenaere-Douyère, «Défendre les intérêts moraux et économiques des inventeurs : des formes d'action collective sous la Révolution (1790-1798) », ARTEFACT, 2015, hors-série, $\mathrm{n}^{\circ} 1$.

(c) Gabriel Galvez-Behar Version de pré-publication/Pre-print version 
Il fut mis en œuvre à de rares occasions et notamment en 1839 pour rendre publique l'invention du daguerréotype jusqu'alors gardée secrète.

Pasteur sollicita ce dispositif particulier à un moment bien précis de sa carrière. En 1872, à l'âge de cinquante ans, il décida de quitter son poste de professeur à la Faculté des sciences mais non pas celui de directeur de laboratoire - en faisant valoir la fatigue causée par de nombreux travaux et par l'hémiplégie qui l'avait frappé quelques années auparavant. Pour justifier l'octroi d'une pension exceptionnelle, il souligna, on l'a vu, le fait que son engagement scientifique ne lui avait pas permis d'assurer à sa famille les conditions d'un avenir serein. Pourtant, au moment même où il entamait cette démarche, loin de se cantonner à un quelconque repos, Pasteur était engagé dans l'exploitation de ses brevets sur la bière. Pasteur put cependant compter sur le soutien du médecin et député Paul Bert qu'il connaissait depuis le milieu des années $1860^{36}$. Dans le rapport qu'il présenta à l'Assemblée, ce dernier eut l'habileté de présenter ce projet de récompense comme « le plus efficace moyen de réparer les erreurs d'une organisation insuffisante » de la science tout en insistant sur les bénéfices économiques des découvertes de Pasteur ${ }^{37}$. Sans discussion, l'Assemblée nationale vota l'attribution d'une pension viagère de 12000 francs qui correspondait globalement au traitement plein d'un professeur de la Faculté des sciences.

À cet abandon des fonctions universitaires correspondit, en fait, une intensification des recherches scientifiques et financières. Lancé à partir de la fin 1876 dans des travaux sur les

36. Dans une lettre adressée à Pasteur, Paul Bert lui déclare : «J'étais allé hier chez vous pour vous entretenir de la loi en question et vous assurer de mon entier et respectueux dévouement en cette affaire comme en toute autre », lettre de Paul Bert à Louis Pasteur, 18 mai 1874, BNF, Papiers Louis Pasteur, NAF 18 101, f 110.

37. Journal officiel de la République française, 12 juillet 1874, p. 4861

(C) Gabriel Galvez-Behar Version de pré-publication/Pre-print version 
maladies contagieuses, Pasteur multiplia les sollicitations : 1200 francs demandés au ministre de l'Instruction publique en juillet 1878, 5000 francs à l'Académie des sciences un mois plus tard, 2500 francs pour des expériences sur la maladie du charbon dans le Jura en septembre de l'année suivante ${ }^{38}$. N'abandonnant pas tout à fait ses recherches antérieures, il fit part à Jacobsen de son souhait de se créer un laboratoire à la campagne afin de continuer ses recherches sur les fermentations :

« Dussè-je, comme Bernard Palissy, brûler mes meubles je suis résolu à me créer cette année un laboratoire à la campagne. N'en ayant pas personnellement les moyens complets, je vais tenter de l'ériger par souscriptions demandées à quelques-uns des sériciculteurs qui profitent directement de l'application de mes études. C'est hardi, mais faisable $»^{39}$

Quelques mois plus tard, en décembre 1879, Pasteur demanda également au ministre de l'Instruction publique le doublement des crédits de son laboratoire de chimie physiologique arguant de «l'extension toujours croissante de ses travaux ${ }^{40}$. Puis, en 1880 , le ministère de l'Agriculture lui fit attribuer un budget annuel de 50000 francs pour financer ses recherches sur la maladie du charbon. L'extension du domaine de Pasteur demandait toujours plus de moyens financiers.

Aussi, lorsque fut lancé un projet de loi visant à augmenter la pension déjà exceptionnelle que Pasteur avait obtenue en 1874, des voix discordantes se firent-elles entendre. Toujours à la manœuvre, Paul Bert avait justifié le passage de 12000 francs à 25000 francs de la pension viagère au nom des découvertes nouvelles que Pasteur avait faites depuis 1874, qu'il s'agisse 38. Ces sollicitations figurent dans la correspondance publiée de Pasteur: L. Pasteur, Correspondance de Pasteur, 1840-1895, op. cit., p. 57-58, 64-65, 110-111.

39. Lettre de Pasteur à Jacobsen, 8 décembre 1878, Ibid., p. 81.

40. Lettre de Pasteur au ministre de l'Instruction publique, 9 décembre 1879, Ibid., p. 121. Le budget devait ainsi passer de 6000 à 12000 francs.

(c) Gabriel Galvez-Behar Version de pré-publication/Pre-print version 
de la découverte des causes de certaines maladies infectieuses, de la vaccination contre la maladie du charbon ou de ses nouvelles recherches sur la rage. Prenant soin d'insister sur les quelque 20 millions de francs d'économies engendrées par le sauvetage d'une bonne partie du cheptel français, Bert excita également l'orgueil national en faisant référence à la récompense décernée à Jenner en Grande-Bretagne. Pourtant, malgré ces arguments, trois députés vinrent remettre en cause le principe d'une récompense. Faisant référence à la production et à la vente des vaccines, le député de l'Aube, Casimir Michou comparait Pasteur à un inventeur qui « passe son temps à poursuivre une découverte, [qui] travaille, fait des dépenses, et quand il a achevé son invention, [...] cherche à en tirer profit. ${ }^{41}$ Assimilé à un simple inventeur, Pasteur était ramené de la science à l'industrie et de l'industrie au profit.

À nouveau l'intervention de Paul Bert permit de faire taire ces critiques que l'écrasante majorité de la Chambre ne voulait pas entendre. Bert reconnut que Pasteur avait été approché par des financiers aux offres alléchantes. Son refus de leurs propositions n'en justifiait que mieux à ses yeux l'octroi d'une nouvelle récompense. Si la loi fut adoptée à la Chambre puis au Sénat, sans réelle discussion, ces critiques nourrirent un soupçon que les adversaires des méthodes pasteuriennes n'hésitèrent pas à exploiter. Ainsi, en 1887, le docteur Lutaud revintil sur cet épisode pour mettre en doute le désintéressement de Pasteur ${ }^{42}$. En fait, Pasteur était pris dans une contradiction. À mesure que son entreprise scientifique se développait et que l'intendance se faisait de plus en plus visible, il était de plus en plus difficile de paraître

41. Journal officiel de la République française. Débats parlementaires. Chambre des députés, 13 juillet 1883 , p. 1707.

42. Auguste Lutaud, M. Pasteur et la rage, Paris, J. Lévy, 1887, p. 405-431.

(C) Gabriel Galvez-Behar Version de pré-publication/Pre-print version 
totalement désintéressé. En outre, l'apport économique des découvertes de Pasteur, qui était l'un des ressorts essentiels de leur reconnaissance, de leur financement et, donc, de leur réalisation, troublait largement l'idée d'une science pure. Seul le recours à un travail de neutralisation à l'œuvre dans les récits de la geste pasteurienne - les rapports de Paul Bert ou L'histoire d'un savant par un ignorant - pouvait en maintenir la fragile illusion. Économique de part en part, la science pasteurienne ne pouvait pas paraître purement désintéressée.

\section{UN SAVANT BREVETÉ}

L'entreprise pasteurienne reposait ainsi sur un désintéressement lucratif, principe d'action grâce auquel l'argent servait de point d'appui à la recherche. Un autre aspect de l'entreprise fut de se situer à la charnière de la science et de l'industrie, de la science pure et de la science appliquée. Même si, là encore, des discours rétrospectifs ont veillé à reconstruire des distinctions alors mises à mal, force est de constater que Pasteur s'appuya sur des dispositifs propres à l'industrie pour défendre ses intérêts. Le brevet d'invention est l'un d'eux et son usage savant est d'autant plus intéressant à étudier qu'il remet en question une autre norme mertonienne de la science selon laquelle la connaissance produite par les acteurs scientifiques doit être mise à disposition de tous. Pourtant, rares sont les travaux qui ont porté une grande attention à ces brevets ${ }^{43}$. Ceux de Maurice Cassier ont toutefois mis en lumière les contradictions de Pasteur qui fit preuve, en la matière, d'un grand pragmatisme, usant de registres de contrôle et de modes d'appropriation variés ${ }^{44}$. Au-delà de l'apport incontestable

43. Voir, par exemple : Claire Salomon-Bayet, Pasteur et la révolution pasteurienne, Paris, Payot, 1986.

44. Maurice Cassier, « Appropriation and commercialization of the Pasteur anthrax vaccine », Studies in History and Philosophy of Science Part C : Studies in History and Philosophy of Biological and Biomedical Sciences, vol. 36, $\mathrm{n}^{\circ}$ 4, 2005, p. 722-742 ; Maurice Cassier, «Producing, Controlling, and Stabilizing 
de ces travaux, il nous faut revenir ici sur ces usages de la propriété industrielle pour mieux se pencher sur l'écriture des brevets, leurs justifications et leur exploitation, autant de moments qui permettent d'éclairer l'activité entrepreneuriale de Louis Pasteur.

\section{$\underline{\text { L'écriture des brevets }}$}

Comme tout autre texte, les brevets ont un lieu social qu'il faut éclairer. Au cours de sa carrière, Louis Pasteur prit en France six brevets d'invention et sept certificats d'addition, soit au total une douzaine de titres de propriété industrielle (tableau 1). N'ayant pas grande signification pris en eux-mêmes, il faut comparer ces chiffres à ceux des brevets pris par les membres de l'Académie des sciences au cours du XIX ${ }^{\mathrm{e}}$ siècle. Une exploration des bases de données des délivrances de brevets français entre 1791 et 1883 permet d'établir que sur les 275 membres de l'Académie des sciences élus avant 1883, 24 furent titulaires de brevets d'invention ${ }^{45}$. Ces derniers se concentrent essentiellement chez les chimistes - la discipline d'origine de Pasteur - et prennent chacun en moyenne deux brevets. Ainsi s'il se rattache aux pratiques d'un groupe d'académiciens - celui des chimistes -, Pasteur s'en démarque par le nombre relativement important de brevets pris durant sa carrière. Il faut ajouter par ailleurs que Pasteur ne fut pas seulement titulaire de brevets français mais aussi, dès les années 1860, de brevets étrangers tant en Belgique qu'en Espagne, en Grande-Bretagne, en Italie ou aux

Pasteur's Anthrax Vaccine : Creating a New Industry and a Health Market », Science in Context, vol. 21, $\mathrm{n}^{\circ}$ 2, 2008, p. 253-278 ; Maurice Cassier, « Louis Pasteur's patents : agri-food Biotechnologies, industry and Public Good », in Jean-Paul Gaudillière, Hans-Jörg Rheinberger (dir.), Living Properties : Making Knowledge and Controlling Ownership in the History of Biology, Berlin, Max Planck Institut für Geschichtswissenschaft, p. 39-49.

45. Ces chiffres sont tirés d'une recherche d'habilitation en cours. Ils reposent sur la fouille de données des bases que l'Institut national de la propriété industrielle a bien voulu mettre à notre disposition.

(C) Gabriel Galvez-Behar Version de pré-publication/Pre-print version 
États-Unis (tableau 2). Dans une certaine mesure, l'usage des brevets par Pasteur est à la fois exceptionnel et normal. 
Tableau 1: Brevets d'invention et certificats d'addition français pris par Louis Pasteur

\begin{tabular}{|c|c|c|c|c|}
\hline Titre & Date de dépôt & Lieu de dépôt & $\begin{array}{l}\text { Caractéristiques de la } \\
\text { description }\end{array}$ & Mandataire \\
\hline $\begin{array}{l}\text { Procédé de fermentation } \\
\text { alcoolique }\end{array}$ & 3 février 1857 & Lille & 24 lignes & Non \\
\hline $\begin{array}{l}\text { Fabrication de l'acide } \\
\text { acétique }\end{array}$ & 9 juillet 1861 & Paris & 16 lignes & Non \\
\hline $\begin{array}{l}\text { Certificat d'addition au } \\
\text { brevet du } 9 \text { juillet } 1861\end{array}$ & $\begin{array}{l}12 \quad \text { décembre } \\
1861\end{array}$ & Paris & 54 lignes & Non \\
\hline $\begin{array}{ll}\text { Procédé relatif à la } \\
\text { conservation des vins }\end{array}$ & 11 avril 1865 & Paris & 9 lignes & Non \\
\hline $\begin{array}{l}\text { Certificat d'addition au } \\
\text { brevet du } 11 \text { avril } 1865\end{array}$ & 27 mai 1865 & Paris & 30 lignes & Non \\
\hline $\begin{array}{l}\text { Certificat d'addition au } \\
\text { brevet du } 11 \text { avril } 1865\end{array}$ & 26 octobre 1865 & Paris & 25 lignes & Non \\
\hline $\begin{array}{l}\text { Procédé de fabrication de } 2 \\
\text { la bière }\end{array}$ & 28 juin 1871 & $\begin{array}{l}\text { Clermont- } \\
\text { Ferrand }\end{array}$ & 24 lignes & Non \\
\hline $\begin{array}{l}\text { Certificat d'addition au } \\
\text { brevet du } 28 \text { juin } 1871\end{array}$ & 4 novembre 1871 & Paris & 40 lignes et un dessin & Non \\
\hline $\begin{array}{l}\text { Certificat d'addition au } \\
\text { brevet du } 28 \text { juin } 1871\end{array}$ & $\begin{array}{ll}25 & \text { novembre } \\
1871 & \end{array}$ & Paris & 102 lignes & $\begin{array}{ll}\text { Non } & \text { mais } \\
\text { écriture } & \\
\text { différente } & \\
\end{array}$ \\
\hline $\begin{array}{l}\text { Certificat d'addition au } \\
\text { brevet du } 28 \text { juin } 1871\end{array}$ & 22 janvier 1872 & Paris & 18 lignes & Non \\
\hline $\begin{array}{l}\text { Certificat d'addition au } \\
\text { brevet du } 28 \text { juin } 1871\end{array}$ & 18 octobre 1872 & Paris & 12 lignes & Non \\
\hline 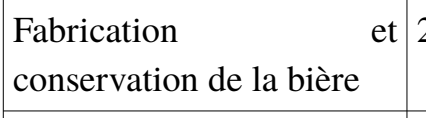 & 21 août 1871 & Paris & 21 lignes & Non \\
\hline 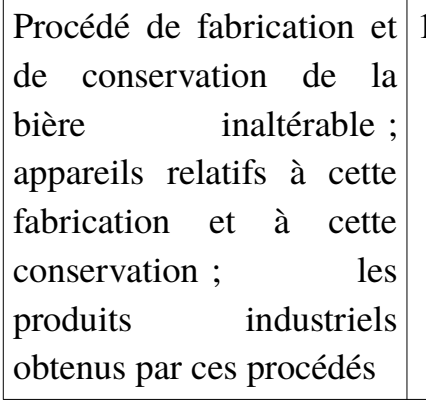 & 13 mars 1873 & Paris & $\mid \begin{array}{l}321 \text { lignes plus un } \\
\text { dessin }\end{array}$ & Paul de Leusse \\
\hline
\end{tabular}

Encore faut-il prêter une attention particulière au contexte de ces demandes. Le premier élément de ce contexte est géographique. Deux des six brevets français l'ont été hors de Paris, 
à Lille, où Pasteur était en poste en 1857, puis à Clermont-Ferrand, où il avait trouvé refuge auprès d'Émile Duclaux du fait de la guerre puis de la Commune en 1871. Or la plupart des brevets français à l'époque étaient délivrés à Paris où il était possible de faire de vraies recherches d'antériorité nécessaires pour évaluer la validité des brevets ${ }^{46}$. Aussi ces deux brevets n'avaient-ils probablement pas tant pour objet principal de revendiquer un droit que de prendre date. L'absence de recours à un ingénieur-conseil vient d'ailleurs corroborer cette hypothèse. Seul le brevet de 1873 est ainsi confié à un mandataire, le comte Paul de Jeusse. Enfin, la rédaction même des brevets est significative. Les brevets sont courts, écrits à la première personne et de la main de Pasteur. Jusqu'à la fin des années 1860 , en tous cas, tout laisserait penser que l'usage fait des brevets par Pasteur ne visait pas prioritairement une exploitation économique.

L'existence de certificats d'addition, qui permettent au titulaire d'un brevet de préciser son invention, vient toutefois nuancer une telle affirmation. Le premier brevet de 1871 , avec ses quatre certificats d'addition étalés sur plus d'une année et demie après la délivrance du brevet initial, marque une rupture que confirment le recours à un mandataire et la prise de brevets à l'étranger. Vu le coût de telles procédures, il est inenvisageable de penser que Pasteur ne pensait pas à une exploitation économique de ses brevets d'invention à partir de cette date. Aussi bien est-ce la finalité de la prise des brevets qui est ainsi interrogée. Pour y voir plus

46. Gabriel Galvez-Behar, La République des inventeurs. Propriété et organisation de l'innovation en France (1791-1922), Rennes, Presses universitaires de Rennes, 2008 ; Jérôme Baudry, « Une histoire de la propriété intellectuelle : les brevets d'invention en France, 1791-1844 », thèse de doctorat d'histoire, École des hautes études en sciences sociales, 2014.

(C) Gabriel Galvez-Behar Version de pré-publication/Pre-print version 
clair, il faut prêter une attention toute particulière aux justifications données par Pasteur et aux critiques auxquelles son rapport à la propriété industrielle donna lieu.

Tableau 2: Brevets de Louis Pasteur pris à l'étranger

\begin{tabular}{|c|c|c|c|}
\hline Pays & Numéro & Date & Titre \\
\hline Bade & & $\begin{array}{l}11 \text { novembre } \\
1873\end{array}$ & $\begin{array}{l}\text { Privilegium für den Grassen Paul de Leusse } \\
\text { zu Reichshoffen }\end{array}$ \\
\hline Belgique & & $\begin{array}{ll}11 & \text { janvier } \\
1862 & \end{array}$ & $\begin{array}{l}\text { Procédé de fabrication du vinaigre ou de } \\
\text { l'acide acétique [in «Brevets accordés en } \\
\text { Belgique d'après les publications faites dans } \\
\text { le Moniteur pendant le mois de janvier », } \\
\text { Bulletin du Musée de l'Industrie, tome 41, } \\
n^{\circ} 2 \text {, février } 1862, \text { p. } 124\end{array}$ \\
\hline Belgique & & 22 mars 1873 & $\begin{array}{l}\text { Procédés nouveaux de fabrication et de } \\
\text { conservation de la bière }\end{array}$ \\
\hline Espagne & 5084 & 25 avril 1873 & $\begin{array}{l}\text { Sistema y nuevos procedimientos para } \\
\text { fabricar y conservar inalterable la cerveza }\end{array}$ \\
\hline États-Unis & 135245 & $\begin{array}{ll}28 & \text { janvier } \\
1873 & \end{array}$ & Improvement in brewing beer and ale \\
\hline États-Unis & 141072 & $\begin{array}{l}22 \text { juillet } 1873 \\
\text { [dépôt le } 9 \text { mai } \\
1873 \text { ] }\end{array}$ & $\begin{array}{l}\text { Improvement in the manufacture of beer and } \\
\text { yeast }\end{array}$ \\
\hline Grande-Bretagne & 2225 & 24 août 1871 & Improvements in brewing \\
\hline Grande-Bretagne & 1106 & 25 mars 1873 & $\begin{array}{l}\text { Improvements in the manufacture of beer } \\
\text { and yeast }\end{array}$ \\
\hline
\end{tabular}

\section{$\underline{\text { Justifier le brevet }}$}

Pour l'hagiographie pasteurienne, la cause est entendue. L'usage que Pasteur fit des brevets n'eut d'autre dessein que de pouvoir mieux faire tomber ses inventions dans le domaine public pour le bénéfice du plus grand nombre au détriment de sa propre fortune. L'un des biographes de Pasteur, Pierre Darmon, affirme ainsi que c'est dans le préambule de son brevet du 9 juillet 
1861 que Pasteur avança un tel principe ${ }^{47}$. Il n'en est rien puisque le dossier original du brevet en question ne comporte guère cette mention qui ne fut introduite, on le verra, que près d'un an plus tard dans un contexte bien particulier ${ }^{48}$. Cette inexactitude est toutefois hautement significative. La légende du brevet laissé volontairement tomber dans le domaine public repose sur une lecture partielle des sources et, de ce fait, sur un oubli du contexte dans lequel il a émergé. Pour mieux comprendre le rapport de Pasteur et de ses interlocuteurs au brevet d'invention, pour mieux saisir la relation difficile entre science et propriété intellectuelle, il faut revenir aux différents contextes.

Si le brevet lillois de 1857 sur la fermentation alcoolique ne semble pas avoir fait l'objet d'une justification particulière, celui de 1861 constitue l'un des moments-clefs de la création de la légende déjà évoquée. Pris le 9 juillet 1861 puis complété par un certificat d'addition le 12 décembre de la même année, le brevet sur la fabrication de l'acide acétique ne fut pas mentionné dans la communication que Pasteur fit à l'Académie des sciences le 10 février $1862^{49}$. En revanche, préparant sa communication de juillet 1862, Pasteur prit un soin particulier à mentionner ce point. Dans l'un de ses brouillons, il écrivait ainsi :

«Comme il arrive fréquemment que des principes scientifiques livrés à la publicité par leurs auteurs deviennent l'objet de brevets d'invention par l'addition parines de dispositifs d'appareils ou de modifications insignifiantes, $\mathrm{j}$ 'ai pris <illisible> pre pris antérieurement à ma [dernière] communication du brevet d'invention qui prime[rait] tous ceux auxquels mon travail pourrait donner lieu. Et jécte aujouth <illisible >illisible $>$ Et je m'empresse d'ajouter que je suis résolu dès aujourd'hui à laisser tomber dans le domaine public le brevet que <illisible $>$ dont je me

47. P. Darmon, Pasteur, op. cit., p. 157.

48. INPI, brevet n ${ }^{\circ}$ 50359, Louis Pasteur, « Fabrication de l'acide acétique », 9 juillet 1861.

49. Louis PASTEUR, «Études sur les mycodermes. Rôle de ces plantes dans la fermentation acétique », Comptes rendus hebdomadaires de l'Académie des sciences, tome 54, 1862, p. 265-270, séance du 10 février 1862

(c) Gabriel Galvez-Behar Version de pré-publication/Pre-print version 
suis muni à la date du 9 juillet 1861 ainsi que l'addition du 22 décembre 1861 de la même année qui y est jointe. $»^{50}$

Il est intéressant de remarquer que ce passage ne fut pas repris dans les Comptes rendus de l'Académie des sciences ${ }^{51}$. Il le fut, en revanche, sous la forme d'une note, dans les colonnes du Moniteur universel qui, dès le lendemain de la communication de Pasteur, publia intégralement son mémoire :

« Comme il arrive fréquemment que des principes scientifiques, livrés à la publicité par leurs auteurs, deviennent entre les mains d'autrui l'objet de brevets d'invention par l'addition de dispositifs d'appareil ou de modifications insignifiantes, j'ai pris antérieurement à ma communication du mois de février, d'après l'avis de personnes autorisées, un brevet qui primerait tous ceux auxquels mon travail aurait pu donner lieu ; et j'ajoute que je suis résolu dès aujourd'hui à laisser tomber ce brevet dans le domaine public $\gg^{52}$

Pasteur faisait ainsi valoir deux principes : le premier visait à lutter contre les profiteurs des travaux scientifiques ; le second soulignait l'intérêt du savant pour le bien commun. Ces deux principes, repris de manière plus ou moins exacte par la geste pasteurienne, doivent toutefois être mis en regard des différences entre les deux versions du texte. En supprimant toute référence précise aux dates de son brevet et de son certificat d'addition, Pasteur procédait à une reconstruction a posteriori de son usage du brevet et tentait d'étouffer toutes les questions sensibles qu'il pouvait susciter : si son intention initiale avait réellement été de faire tomber son brevet dans le domaine public, pourquoi avait-il attendu pour le faire une année, jour pour jour, à la veille de payer la seconde annuité de son brevet ? Pourquoi avait-il pris un certificat

50. BNF, Papiers Louis Pasteur, NAF 18091, fo 37, sd.

51. Louis PASTEUR, «Suite à une précédente communication sur les mycodermes. Nouveau procédé industriel de fabrication du vinaigre », Comptes rendus hebdomadaires de l'Académie des sciences, tome 55, Paris, Gauthier-Villars, 1862, p. 28-32, séance du 7 juillet 1862.

52. Le Moniteur universel, 8 juillet 1862, p. 1029.

(C) Gabriel Galvez-Behar 
d'addition ? Pourquoi n'avait-il pas évoqué son brevet pris en Belgique ? Toutes ces questions

pouvaient jeter une ombre sur le désintéressement affiché du savant.

Des doutes furent d'ailleurs exprimés dès la publication du Moniteur universel et de la brochure que Pasteur avait fait éditer en juillet $1862^{53}$. Dans sa livraison du $1^{\text {er }}$ août, le Moniteur scientifique signalait la communication de Pasteur à l'Académie de manière cinglante :

« Nous insérerons ce travail de M. Pasteur dans nos comptes-rendus de chimie. Disons en attendant que $\mathrm{M}$ Pasteur fait abandon à l'industrie du brevet qu'il avait eu la malheureuse idée de prendre. C'est 100 francs, prix d'une annuité, que lui aura coûté la fantaisie de vouloir ajouter à son titre de directeur de l'École normale celui de fabricant de vinaigre $»^{54}$

Sous la plume du docteur Quesneville, le brevet de Pasteur constituait un malheureux mélange des genres dont la justification était douteuse. La Revue viticole fut quant à elle plus clémente. Claude Ladrey, professeur de chimie à la faculté des sciences de Dijon, salua ainsi l'initiative de Pasteur :

«Un brevet pris avant le mois de février empêchera l'usurpation de ces industriels que l'on voit trop souvent venir, par une modification insignifiante, s'approprier et confisquer à leur profit des inventions qu'ils n'ont pas faites, et empêcher pendant de longues années l'extension et le développement d'une idée qui ne leur appartenait pas.

Après avoir pris cette utile précaution, M. Pasteur abandonne son brevet et le laisse tomber dans le domaine public. Nous le félicitons bien sincèrement d'avoir compris que la science, pour conserver son prestige et sa force, doit rester étrangère à la spéculation, qui, souvent, conduit à des actes dont le souvenir reste comme une tache sur la mémoire des noms respectés. ${ }^{55}$

53. Louis Pasteur, Nouveau procédé industriel de fabrication du vinaigre, Paris, Mallet-Bachelier, 1862.

54. Le Moniteur scientifique, $\mathrm{n}^{\circ} 135,1^{\mathrm{er}}$ août 1862, p. 492.

55. Revue viticole, 1862 , p. 315.

(C) Gabriel Galvez-Behar

Version de pré-publication/Pre-print version 
Mais, tout en félicitant Pasteur pour son geste, le rédacteur de la Revue viticole envoyait en même temps un avertissement à tous ses collègues. Exploiter un brevet eût constitué une transgression.

Ce point de vue n'était cependant pas unanime. Charles-Louis Barreswil, professeur de chimie industrielle à l'École Turgot puis à l'École supérieure de commerce, affirmait ainsi :

« Ce procédé, essentiellement pratique et suivi sur une échelle industrielle, a été breveté par M. Pasteur. Le Moniteur, qui a reproduit in extenso le mémoire de l'auteur, explique que M. Pasteur n'a pris ce brevet que pour empêcher un tiers d'en prendre un, et de priver ainsi le domaine public du fruit de son travail. Je ne sais pas pourquoi M. Pasteur ne prendrait pas un brevet pour assurer à sa peine une très légitime rémunération [...]. Quoi qu'il en soit, dès que l'intention de M. Pasteur est d'assurer au domaine public les droits qu'eût pu lui conférer son brevet, ce savant a pris, en demandant un brevet, le parti le meilleur. Autrement un brevet parasite eût jeté [...] le grappin destiné à prendre ce qu'il eut (sic) été appelé une expérience de laboratoire ${ }^{56}$

Non seulement Barreswil jugeait légitime l'exploitation économique d'un brevet par un savant mais il s'associait à la lutte de Pasteur contre les brevets parasites. L'expression d'expérience de laboratoire renvoyait, en effet, à une série de décisions judiciaires intervenues à partir des années 1850 et qui considéraient qu'une expérience scientifique ne pouvait pas constituer une antériorité susceptible d'invalider un brevet pour défaut de nouveauté ${ }^{57}$. D'ailleurs, au moment même où Pasteur publiait sa brochure, le Tribunal civil de la Seine jugeait l'affaire dite de la fuchsine ${ }^{58}$. Or certaines parties au procès tentaient d'utiliser les travaux du chimiste allemand Hoffmann pour invalider certaines brevets en cause. Par son

56. [Charles-Louis Barreswil], « Nouveau procédé industriel de fabrication du vinaigre par M. L. Pasteur », Revue de chimie appliquée, juillet 1862, p. 279-280.

57. Adrien Huard, Répertoire de législation et de jurisprudence en matière de brevets d'invention, Paris, Cosse et Marchal, 1863, p.483-485.

58. Henk Van Den Belt, "Why Monopoly Failed : The Rise and Fall of Société La Fuchsine », The British Journal for the History of Science, vol. 25, $\mathrm{n}^{\circ}$ 1, 1992, p. 45-63.

(c) Gabriel Galvez-Behar Version de pré-publication/Pre-print version 
jugement du 9 août 1862, le tribunal considéra que les travaux scientifiques antérieurs ne pouvaient pas être considérés comme des résultats industriels et que les savants chimistes ne sauraient être regardés comme les inventeurs du colorant qui était en cause. Le tribunal ajouta ainsi :

«En vain on prétend qu'une pareille interprétation tendrait à dépouiller la science au profit de l'industrie ; que cette distinction est dans la loi et dans la nature des choses ; que la science tend à développer les connaissances utiles, à faire progresser les arts et l'industrie ; qu'en chimie surtout, elle fait souvent des observations et des constatations sans s'occuper des résultats industriels qu'elle pourrait produire, ne s'y arrêtant pas, ne les formulant pas, ne les complétant pas, ouvrant la porte à tous, et trouvant sa gloire dans les avantages que les autres en retirent ; que l'industrie, au contraire, se borne à produire, profitant des voies qui lui sont ouvertes par la science, et offrant à la société les résultats que la loi sur les brevets a pour objet de protéger uniquement. ${ }^{59}$

Ce contexte économico-juridique fait apparaître le recours aux brevets et sa justification par Pasteur sous un autre angle. Si cette dernière s'affiche comme une preuve du désintéressement du savant, elle constitue dans le même temps un refus d'une distinction préjudiciable à la science, qui devrait se payer de gloire tandis que l'industrie pourrait librement tirer profit de ses travaux. Le désintéressement pasteurien se présente ainsi comme un désintéressement actif où le savant prend la précaution de préserver ses droits économiques aussi longtemps qu'il n’a pas décidé de ne pas les abandonner. C'est à lui que revient la décision de rester cantonné dans la sphère de la science «pure » ou de s'impliquer dans l'exploitation économique de ses découvertes. Il n'y a donc pas un rejet définitif de cette dernière mais une tension que Pasteur assume et qui constitue, en fait, le ressort de son entreprise scientifique.

59. La Presse, 27 septembre 1862, p. 3. 
Aux mains de Pasteur, le brevet d'invention s'avère être un instrument relevant aussi bien du champ scientifique que du champ industriel. Moyen de faire valoir ses droits auprès des fabricants, il peut aussi être utilisé pour s'imposer auprès de collègues dans le cadre d'un conflit de priorité. Quelques années plus tard, en 1865, un nouveau brevet sur la conservation des vins fit ainsi l'objet une justification supplémentaire. Alors qu'il avait entamé une collaboration avec plusieurs viticulteurs et avec Vergnette de Lamotte sur les procédés de chauffage du vin, Pasteur prit un brevet le 11 avril 1865. Trois semaines plus tard à l'Académie des sciences, le $1^{\text {er }}$ mai, alors que Vergnette avait transmis une communication sur le sujet, Pasteur intervint et évoqua son brevet en ces termes :

«L'Académie comprendra qu'il faille attendre plusieurs années pour juger un tel procédé dans son application industrielle parce que le vin met souvent un temps considérable à devenir malade. Aussi mon intention n'était pas de faire de longtemps une publication académique à ce sujet. Je me suis borné, afin de prendre date, à une publicité dont j'ai déjà usé, et qui laisse au savant toute sa liberté d'esprit et d'action dans les recherches de cette nature, je veux parler de la demande d'un brevet d'invention. ${ }^{60}$

Selon Pasteur, le brevet de 1865 avait donc pour seul but de faire valoir, au besoin, une priorité sans pour autant avoir une dimension économique quelconque. Ce fut d'ailleurs ce qu'il déclara à son interlocuteur, Vergnette de Lamotte, dans une lettre de juillet 1875 :

« Vous aurez remarqué, dans le compte rendu du $1^{\mathrm{er}}$ mai, que $\mathrm{j}$ 'avais pris un brevet d'invention, dans le but principalement de suivre à mon aise l'application de ce moyen, et voilà que je suis tourmenté pour en profiter, ce qui me répugne pour toutes sortes de motifs. Ma femme, qui seule compte dans le ménage, et que l'avenir de ses enfants préoccupe, me donne les meilleures raisons du monde en apparence pour lever mes scrupules. Je ne sais même pas si elle ne devait pas vous en écrire et prendre conseil de votre expérience, voire même vous proposer une association $! »^{61}$

60. Comptes rendus hebdomadaires de l'Académie des sciences, t. LX, $\mathrm{n}^{\circ} 18,1865$, p. 899-900, séance du $1^{\mathrm{er}}$ mai 1865.

61. Lettre de Pasteur à Vergnette de Lamotte, 11 juillet 1865, citée in Louis Pasteur, « Lettre à M. le Directeur du Journal d'agriculture pratique », Journal d'agriculture pratique, vol. 36, n² 2, 1872, p. 92. Nous n'avons pas

(C) Gabriel Galvez-Behar Version de pré-publication/Pre-print version 
Toutefois, on mesure bien l'ambiguïté créée par Pasteur qui, tout en se défendant de vouloir exploiter son brevet, suggère qu'une association pourrait intéresser sa femme, garante de l'économie du ménage. On notera, également, le glissement entre la justification donnée à l'Académie, où Pasteur fait du brevet un moyen de publicité pour prendre date, et celle donnée à Vergnette. En fait, ces deux justifications produites à quelques mois d'écart étaient déjà empreintes de contradictions. Les brevets d'invention, qui n'étaient délivrés que chaque trimestre - mais entrant en vigueur à la date de la demande -, n'étaient guère disponibles sous forme publiée. Consultables au ministère du Commerce dès leur délivrance, les brevets ne furent systématiquement publiés dans leur intégralité qu'à partir de 1902. Aussi la justification donnée par Pasteur pouvait paraître d'autant moins convaincante qu'il lui était toujours possible de prendre date d'une autre manière, par un pli cacheté ou par la publication d'une lettre dans une revue quelconque.

À l'automne 1865, Pasteur usa de son brevet comme d'une preuve de la priorité de sa découverte. Dans une lettre envoyée le 11 octobre au Moniteur viticole, qui avait entretemps souligné les mérites de Vergnette-Lamotte, il évoqua en ces termes la note de ce dernier, lue le $1^{\text {er }}$ mai 1865 à l'Académie des sciences :

« Relativement à cette note et au point de vue de priorité de mon procédé, le doute n'est pas permis, puisque j'avais pris date trois semaines auparavant (11 avril 1865) par un brevet d'invention, dans le but de pouvoir suivre à mon aise toutes mes expériences et correspondre avec un grand nombre de personnes, sans craindre que l'on me devançât par quelque publication ou brevets. $»^{62}$

retrouvé la minute de cette lettre, que Pasteur disait avoir conservée, dans les papiers Pasteur de la BNF. Son contenu n'est cependant pas remis en question par Vergnette de Lamotte dans son conflit qui l'oppose alors à Pasteur. 
Là encore intervenait un nouveau glissement : non seulement Vergnette devenait celui à qui l'on pouvait opposer le brevet mais encore ce dernier apparaissait comme un moyen de continuer à « correspondre avec un grand nombre de personnes ». Le brevet apparaissait ainsi comme une précaution nécessaire pour celui qui était impliqué dans une large collaboration avec des viticulteurs qui pourraient avoir un intérêt à devancer Pasteur. Aussi est-ce bien le type de science entreprise par Pasteur qui nécessitait une forme de protection particulière que ne pouvaient pas fournir les dispositifs propres au champ scientifique.

Vergnette-Lamotte protesta et la querelle s'envenima rapidement. Elle dura plus sept années pendant lesquelles la question du brevet ne cessa d'être au cœur des discussions. En 1866, Le Moniteur scientifique contesta l'usage que Pasteur faisait du brevet et lui opposa un avis émis par l'Académie des sciences pour qui « la priorité appartient incontestablement à celui qui, le premier, a livré ses observations à la publicité. ${ }^{63}$ Or, souligna Le Moniteur scientifique, un brevet n'étant pas délivré au moment de sa demande, on ne pouvait pas utiliser la date de cette dernière dans une querelle de priorité ${ }^{64}$. La remarque était d'autant plus justifiée que le brevet n'était pas facilement à la disposition du public. En 1869, l'agronome et académicien Paul Thénard, soutien de Vergnette dans la querelle, intima d'ailleurs à Pasteur

62. Lettre de Pasteur au Moniteur viticole, 11 octobre 1865, citée in Louis Pasteur, Études sur le vin : ses maladies, causes qui les provoquent, procédés nouveaux pour le conserver et pour le vieillir, Paris, Imprimerie impériale, 1866, p. 230.

63. Comptes rendus hebdomadaires de l'Académie des sciences, t. 34, 1852, p. 830. Si, contrairement à ce qu'affirme le rédacteur du Moniteur scientifique, ces lignes ne sont pas du seul Arago, elles font écho à un principe qu'Arago avait déjà affirmé dès les années 1840 à propos de la querelle sur la découverte de Neptune.

64. Le Moniteur scientifique, 15 août 1866, p. 708.

(C) Gabriel Galvez-Behar Version de pré-publication/Pre-print version 
de publier son brevet de 1865 , ce que fit Pasteur dans une note adressée à l'Académie des sciences en octobre $1869^{65}$.

La controverse ne s'éteignit pas pour autant. Elle se poursuivit en 1872 lorsque Pasteur fit l'objet de critiques - voire d'attaques - de la part du chimiste et académicien Edmond Frémy qui contestait les travaux pasteuriens sur les fermentations. À nouveau, la question du brevet fut évoquée. Le chimiste et académicien Antoine-Jérôme Balard, qui n'avait de cesse que de défendre Pasteur, rappela la priorité qu'instituait le brevet du 11 avril 1865 et justifia la prise de ce dernier ainsi :

« Ce brevet fut pris par M. Pasteur, quand ses idées sur la conservation des vins furent bien arrêtées, afin de se mettre en garde contre les frelons de l'industrie. Il l'a laissée volontairement tomber dans le domaine public, et ceux qui parlent avec un certain dédain de cette manière d'assurer la propriété d'une découverte industrielle peuvent en faire dès lors usage gratuitement. ${ }^{66}$

Par ces quelques lignes Balard reprenait certes l'argumentation de Pasteur sur un point - la protection contre les «frelons de l'industrie »- mais il la modifiait sur deux autres. En 1865, on l'a vu, Pasteur avait présenté son brevet comme une manière de prendre date et d'anticiper une publication scientifique qui aurait nécessité plusieurs années de travaux. Sous la plume de Balard, loin d'être une publication de second ordre, le brevet avait été pris quand les idées de Pasteur avaient été «bien arrêtées ». Il apparaissait ainsi comme une pièce tout à fait recevable dans la querelle de priorité. En outre, Balard reprenait la doctrine du brevet tombé

65. Comptes rendus hebdomadaires de l'Académie des sciences, t. 69, 1869, p. 749, séance du 13 septembre 1869 ; réponse de Pasteur in Comptes rendus hebdomadaires de l'Académie des sciences, t. 69, 1869, p. 905911, séance du 25 octobre 1869.

66. Comptes rendus hebdomadaires de l'Académie des sciences, t. 74, 1872, p. 566, séance du 26 février 1872.

(C) Gabriel Galvez-Behar Version de pré-publication/Pre-print version 
dans le domaine public, doctrine qui avait déjà été ébauchée en 1862 et qui avait le mérite d'insister sur le désintéressement de Pasteur ${ }^{67}$.

Peut-être cela incita-t-il Vergnette-Lamotte à faire valoir un autre argument dans les colonnes du Journal d'agriculture pratique. Tout en affirmant que les « revendications de priorité [n'avaient] aucun intérêt», Vergnette-Lamotte y déclarait que «M. Pasteur ne [pouvait] avoir oublié qu'il eut, en 1865, la pensée d'exploiter son brevet, en s'associant avec moi, comme il [...] le donnait à entendre dans sa lettre du 13 juillet $»^{68}$. En évoquant ainsi un projet d'association, Vergnette-Lamotte mettait ainsi à mal la doctrine du domaine public reprise quelques mois plus tôt. Aussi Pasteur réagit-il violemment à cette lettre en la replaçant tout d'abord sur le terrain des questions de priorité « qui intéressent la moralité publique, parce qu'elles traitent de la propriété scientifique, plus respectable encore que toute autre propriété. ${ }^{69}$ Faisant écho aux intenses débats sur la propriété intellectuelle qui avaient alors lieu, Pasteur considérait que la science et ses savants étaient en droit d'avoir des droits au même titre que les artistes ou les inventeurs ${ }^{70}$. Pour autant, Pasteur écartait l'idée d'avoir voulu s'associer à Vergnette et, publiant la minute de la lettre du 13 juillet, il laissa entendre qu'un tel projet n'avait été qu'inspiré par son épouse ${ }^{71}$. Quelques semaines plus tard, cependant, Pasteur se fit moins catégorique en affirmant :

67. Cet argument est repris par le confrère et ami de Pasteur Henri Sainte-Claire Deville en 1869 Comptes rendus hebdomadaires de l'Académie des sciences, t. 69, 1869, p. 1050.

68. Journal d'agriculture pratique, 11 juillet 1872, p. 54.

69. Journal d'agriculture pratique, 18 juillet 1872, p. 91.

70. Gabriel Galvez-Behar, "The Propertisation of Science : Suggestions for an Historical Investigation », Comparativ. Zeitschrift für Globalgeschichte und vergleichende Gesellschaftsforschung, vol. 21, ${ }^{\circ}$ 2, 2011, p. $80-97$.

71. $C f$ supra.

(C) Gabriel Galvez-Behar Version de pré-publication/Pre-print version 
«Je ne vois pas en quoi une proposition d'association aurait affirmé que M. Vergnette avait une part dans la démonstration expérimentale de l'efficacité de la méthode du chauffage. Si je venais à proposer une association à l'un des grands industriels qui exploitent mon procédé de fabrication du vinaigre ou mon procédé de confection de la graine de vers à soie, cela signifierait-il que je leur reconnais une part dans la découverte de ces procédés ? ${ }^{72}$

L'absence de distinction entre le potentiel et l'irréel permettait à Pasteur de créer une ambiguïté laissant croire, à qui voudrait bien l'entendre, qu'il n'avait eu aucune idée d'association en tête. Eût-il toutefois évoqué d'autres de ses travaux et non pas ceux sur le vinaigre ou les vers à soie que le sens de sa phrase en eût été changé ? Car, au moment même où son brevet de 1865 était justifié par son désintéressement et sa volonté d'enrichir le domaine public, Pasteur était largement impliqué dans l'exploitation de son brevet sur la bière.

\section{Tirer profit}

Si jusqu'alors le recours aux brevets d'invention par Pasteur relève d'une stratégie ambiguë, les brevets sur la bière pris en 1871 et en 1873 firent très clairement l'objet d'une exploitation commerciale dans laquelle Pasteur fut impliqué. Malgré la discrétion de ce dernier et la rareté des sources, il est possible d'établir clairement cette dimension économique des brevets pasteuriens sur la bière. Leur longueur, le grand nombre de certificats d'addition dont ils firent l'objet et, surtout, leurs répliques à l'étranger avec une demidouzaine de brevets en Espagne, aux États-Unis, en Grande-Bretagne ou en Italie témoignent déjà du statut particulier de ces brevets. La correspondance avec le chimiste Jean-Baptiste Dumas, alors secrétaire perpétuel de l'Académie des sciences, vient quant à elle lever toute équivoque.

72. Journal d'agriculture pratique, $1^{\mathrm{er}}$ août 1872, p. 164.

(C) Gabriel Galvez-Behar Version de pré-publication/Pre-print version 
Ces études sur la bière avaient été entamées en 1871 dans le laboratoire de Duclaux à Clermont-Ferrand où Pasteur prit son premier brevet sur la bière le 28 juin 1871 - la bière dite de « la Revanche »- et c'est depuis Clermont qu'il mit régulièrement Dumas au courant de ses recherches. Dès la mi-juillet, Pasteur écrivait à ce dernier et évoquait les brevets pris et à prendre :

« Je vous remercie beaucoup de vos offres et de vos conseils ; je pense que je pourrai faire moi-même les dépenses des brevets, même celui pour l'Angleterre ; mais ensuite que faire et comment en tirer profit ? Vos conseils pourront m'être très utiles. ${ }^{73}$

De manière très directe, Pasteur avouait donc vouloir « tirer profit » de ses brevets et faisait appel aux conseils de Dumas, dont la stature et les liens avec les milieux économiques pouvaient être utiles. Si nous ignorons quelle fut la nature des conseils de Dumas puisque la correspondance passive de Pasteur déposée à la Bibliothèque nationale ne comprend pas les lettres de cet échange, la suite du courrier de Pasteur confirme bien la portée que Pasteur entend donner à sa nouvelle découverte :

« Quant à mes garanties, voici celle que j'ai : j'ai pris un brevet pour la France et j'ai écrit en Autriche à M. Chiozza, riche propriétaire d'Illyrie, et dont le nom, comme chimiste, doit vous être connu, pour qu'il prenne en mon nom des brevets en Autriche, en Italie et en Amérique. Je pense que je peux attendre mon retour à Paris pour ceux d'Angleterre, de Belgique et d'Allemagne. ${ }^{74}$

Deux semaines plus tard, Pasteur envoya son brevet français à Dumas et lui rappela son intention de prendre des brevets à l'étranger ${ }^{75}$. Pasteur avait fait connaissance de Luigi Chiozza lors d'un séjour en Italie où il avait été appelé, à la demande du gouvernement italien,

73. Lettre de Pasteur à Dumas, 16 juillet 1871, Archives de l'Académie des sciences, Fonds Dumas, J.B.D. 81.

74. Idem

75. Lettre de Pasteur à Dumas, $1^{\text {er }}$ août 1871, Archives de l'Académie des sciences, Fonds Dumas, J.B.D. 81. Cette indication, qui figure dans une note en marge de la lettre, est, encore une fois, omise par la correspondance publiée de Pasteur. L. Pasteur, Correspondance de Pasteur, 1840-1895, op. cit., p. 543-544. 
à travailler sur les vers à soie. Pendant la guerre franco-prussienne, Chiozza, chimiste, industriel et député, était intervenu auprès du gouvernement italien afin que fût proposée à Pasteur une chaire à l'Université de Milan ou à Pise ${ }^{76}$. Dès son retour à Paris, Pasteur fit ainsi établir des procurations à son intention et d'autres, en blanc, pour des brevets à prendre en Belgique, en Prusse et même en Égypte ${ }^{77}$. Dans le contexte troublé de l'effondrement de la France, Pasteur avait ainsi entrepris de se lancer dans l'exploitation de ses brevets sur la bière.

Le recours aux brevets par Pasteur ne fut ainsi ni gratuit, ni encore moins fortuit. Il intervint à des moments-clefs de ses travaux, inaugurant, pour ainsi dire, plusieurs séquences de recherche majeures. Les brevets permirent ainsi à Pasteur de prendre date à la fois dans le champ scientifique et dans le champ industriel alors même que ses recherches remettaient en cause cette distinction instable. Dans le premier, ils étaient une manière de faire valoir une priorité dans le débat scientifique ; dans le second ils devaient permettre de faire valoir, le cas échéant, des droits auprès des industriels. Appartenant à cette longue tradition de la chimie industrielle, incarnée entre autres par Dumas, Pasteur utilisa le brevet pour marquer le champ de ses propres recherches, lui-même situé dans cette zone de contact entre la science et l'industrie. Alors que sur le plan juridique était introduite une séparation de plus en plus nette entre la première et la seconde - l'industrie pouvant construire des fortunes aux dépens de la science qui devait se contenter de la gloire -, Pasteur se servit du brevet pour contrôler les ressorts de cette distinction voire pour la retourner à son avantage.

76. Maurice Vallery-Radot, Pasteur, op. cit., p. 357-358.

77. Répertoire XLIII, procurations des 10, 11, 18, 25 août 1871 ; répertoire LXIII, procuration du 4 septembre 1871. Les procurations n'ont pas pu être retrouvées dans les minutes de l'étude.

(C) Gabriel Galvez-Behar Version de pré-publication/Pre-print version 
Ainsi, si la légende du brevet tombé dans le domaine public peut s'accorder avec le sort des tout premiers brevets de Pasteur, elle ne saurait en rien rendre compte de l'exploitation économique des derniers brevets qui invite, dès lors, à s'interroger sur les liens entre Pasteur et le capital.

\section{PASTEur, UN CAPITAliste PARMi les SAVANTS ?}

Non seulement l'exploitation des brevets sur la bière met à mal la légende du brevet tombé dans le domaine public mais elle éclaire de manière extrêmement fine la collaboration de Pasteur avec le monde de la finance de son temps. Dès lors, la participation de Pasteur au conseil d'administration du Crédit foncier ou encore la création initiale de l'Institut Pasteur sous la forme d'une société anonyme n'apparaissent plus comme des épiphénomènes.

\section{Pasteur, créateur d'entreprise}

Bien qu'ils nous soient fort mal connus, les premiers temps de l'exploitation des brevets sur la bière ont laissée des traces encore inexploitées. Juste après avoir pris son brevet anglais daté du 24 août 1871 - Pasteur partit pour l'Angleterre mener des expériences dans différentes brasseries de la région de Londres. Peu de sources nous renseignent, en revanche, sur la réalisation des projets pasteuriens durant l'année 1872. Au début de l'année 1873, Pasteur continuait à informer Dumas de ses projets. Dans une lettre du 5 janvier 1873, il l'informa de ses tractations avec Isaac Pereire et son fils Eugène qui envisageaient de créer une grande brasserie et d'exploiter les brevets Pasteur dans l'Europe entière ${ }^{78}$. Avec Dumas, Pasteur convint cependant que ces pourparlers devaient rester discrets ${ }^{79}$. S'agissait-il de protéger 78. Lettre de Pasteur à Dumas, 5 janvier 1873, AS, Fonds Dumas, JBD 93.

79. Lettre de Pasteur à Dumas, 16 février 1873, AS, Fonds Dumas, JBD 93.

(C) Gabriel Galvez-Behar Version de pré-publication/Pre-print version 
l'intégrité du savant qui était déjà en train de solliciter une mise à la retraite anticipée ? Ou n'est-ce pas plutôt que Pasteur menait deux négociations en parallèle ?

L'acte fondateur de la Société des bières inaltérables du 4 mars 1873 donne une réponse à ces questions $^{80}$. Si le préambule du document présentait cette société comme le résultat d'une sollicitation exprimée par plusieurs «capitalistes amis» de Pasteur, ce dernier était formellement le fondateur de cette société de vingt ans au capital de 250000 francs. Les 19 actionnaires de la société étaient cependant largement dominés par les banquiers et, plus particulièrement, par les représentants du groupe du Crédit foncier et du Crédit agricole ${ }^{81}$. Cette forte présence de ces deux banques, dont les dirigeants s'étaient opposés aux Pereire sous le Second Empire, confirme l'idée selon laquelle les tractations avec les Pereire n'étaient qu'une hypothèse parmi une autre. Elle éclaire également le rôle de Dumas, qui ne fut pas seulement le mentor de Pasteur dans cette affaire mais qui joua probablement un rôle d'intermédiaire actif du fait de son statut d'administrateur à la fois du Crédit foncier et du Crédit agricole. Georges de Soubeyran, sous-gouverneur du Crédit foncier et du Crédit agricole, député bonapartiste, était l'actionnaire principal, apportant $20 \%$ du capital. Des liens familiaux renforçaient, par ailleurs, la cohérence du groupe : Évariste Robert de Beauchamp était le beau-frère de Soubeyran tandis que Frédéric et Jules Reiset, fils de Jacques Reiset, ancien régent de la Banque de France, étaient les beaux-frères de Charles

80. AN, MC/ET/LIX/709, Statuts de la Société anonyme La Société des Bières inaltérables, procédé Pasteur, 4 mars 1873.

81. Nicolas Stoskopf, Les patrons du Second Empire. Banquiers et financiers parisiens, Paris, France, Picard, 2002, p. 43 ; Jean-Pierre Alline, Banques et bâtisseurs. Un siècle de Crédit foncier, 1852-1884, Paris, Éditions du CNRS, 1994.

(C) Gabriel Galvez-Behar Version de pré-publication/Pre-print version 
Adrien Lebègue de Germiny, lui-même fils de Charles de Germiny, ancien gouverneur de la

Banque de France et du Crédit foncier. La Société des bières inaltérables créée par Pasteur était ainsi soutenue par des banquiers dont le rôle s'était affirmé sous le Second Empire grâce à leur proximité avec le régime.

Tableau 3: Liste des actionnaires de la Société des bières inaltérables au 5 mars 1873 (AN, MC/ET/LIX/709 et Almanach national, Paris, Berger-Levrault, 1873)

\begin{tabular}{|c|c|c|}
\hline $\begin{array}{l}\text { Nom } \\
\text { (en gras figurent les } \\
\text { administrateurs) }\end{array}$ & Qualité & $\begin{array}{l}\text { Montant } \\
\text { souscrit (en } \\
\text { F.) }\end{array}$ \\
\hline $\begin{array}{lr}\text { Soubeyran } & (\text { Jean } \\
\text { Marie } & \text { Georges, } \\
\text { baron de }) & \end{array}$ & $\begin{array}{l}\text { Membre de l'Assemblée nationale - Député de la Vienne } \\
\text { (bonapartiste) - Sous-gouverneur du Crédit foncier et du } \\
\text { Crédit agricole }\end{array}$ & 50000 \\
\hline $\begin{array}{l}\text { Lebègue (Charles } \\
\text { Adrien comte de } \\
\text { Germiny) }\end{array}$ & $\begin{array}{l}\text { Trésorier payeur général de la Seine-inférieure - Régent de la } \\
\text { Banque de France Membre des conseils d'administration du } \\
\text { Crédit foncier et du Crédit agricole }\end{array}$ & 25000 \\
\hline Pasteur (Louis) & Membre de l'Institut & 25000 \\
\hline $\begin{array}{l}\text { Schnapper (Antoine } \\
\text { Maurice) }\end{array}$ & $\begin{array}{l}\text { Banquier - Membre du Conseil d'administration de la Banque } \\
\text { de Paris et des Pays Bas }\end{array}$ & 25000 \\
\hline $\begin{array}{l}\text { Artus (François } \\
\text { Marie, marquis de } \\
\text { Scepeaux) }\end{array}$ & $\begin{array}{l}\text { Propriétaire - Actionnaire fondateur de la Banque de Paris et } \\
\text { des Pays Bas }\end{array}$ & 20000 \\
\hline $\begin{array}{l}\text { Beauchamp (Louis } \\
\text { Évariste Robert de) }\end{array}$ & $\begin{array}{l}\text { Propriétaire - Membre des conseils d'administration du Crédit } \\
\text { foncier et du Crédit agricole }\end{array}$ & 20000 \\
\hline $\begin{array}{l}\text { Leusse (Louis Paul, } \\
\text { comte de) }\end{array}$ & Propriétaire - Ancien député du Bas-Rhin & 15000 \\
\hline Haber (Samuel de) & Banquier-Censeur de la Banque de Paris et des Pays-Bas & 10000 \\
\hline Reiset (Jules) & $\begin{array}{l}\text { Membre du Conseil général de la Seine-inférieure - } \\
\text { Correspondant de l'Académie des sciences }\end{array}$ & 10000 \\
\hline $\begin{array}{l}\text { Ayguevives } \\
\text { (Jacques Auguste, } \\
\text { comte de) }\end{array}$ & $\begin{array}{l}\text { Propriétaire - Membre du conseil d'administration du Crédit } \\
\text { agricole - Conseiller général de la Haute-Garonne }\end{array}$ & 5000 \\
\hline Brazon (Armand) & Propriétaire - Chef de division-adjoint au Crédit foncier & 5000 \\
\hline Burns (Walter) & Propriétaire & 5000 \\
\hline
\end{tabular}




\begin{tabular}{|l|l|l|}
\hline Haber (Adolphe de) & Banquier & 5000 \\
\hline $\begin{array}{l}\text { Lenormand } \\
\text { (Ariste) }\end{array}$ & Propriétaire & 5000 \\
\hline $\begin{array}{l}\text { Pillet Will (Frédéric, } \\
\text { comte) }\end{array}$ & $\begin{array}{l}\text { Propriétaire - Régent de la Banque de France } \\
\text { Vice-secrétaire de la Caisse d'épargne et de prévoyance de } \\
\text { Paris }\end{array}$ & 5000 \\
\hline Reiset (Frédéric) & Conservateur des musées nationaux & 5000 \\
\hline $\begin{array}{l}\text { Sugny (Francisque } \\
\text { de) }\end{array}$ & $\begin{array}{l}\text { Membre de l'Assemblée nationale - Député de la Loire } \\
\text { (légitimiste) }\end{array}$ & 5000 \\
\hline Walsh (vicomte) & Propriétaire & 5000 \\
\hline Waru (Adolphe de) & Propriétaire - Ancien régent de la Banque de France & 5000 \\
\hline
\end{tabular}

La création de cette société permettait à Pasteur d'apporter à la société son brevet du 28 juin 1871, les certificats d'addition afférents ainsi que les brevets pris en Angleterre, en Autriche, en Italie et en Belgique ainsi que « les bénéfices à provenir des licences déjà concédées jusqu'à ce jour », preuve qu'une première exploitation du brevet de 1871 était déjà en cours $^{82}$. Par ailleurs, toute découverte de Pasteur relative à la bière appartiendrait à la société qui recevait également le droit de prendre, le cas échéant, de nouveaux brevets ${ }^{83}$. Enfin, Pasteur s'engageait « à donner son concours à la mise en œuvre de ses procédés et à la surveillance de leur application durant toute la durée des brevets ». Pour cela, il lui était possible de s'adjoindre des chimistes choisis et rétribués par lui-même ${ }^{84}$.

Le prix de cet apport et de ces engagements n'était pas négligeable. Pasteur recevait non seulement une somme de 150000 francs mais en outre vingt actions de fondateur lui donnant

82. AN, MC/ET/LIX/709, Statuts de la Société anonyme La Société des Bières inaltérables, procédé Pasteur, 4 mars 1873, art. 4. Il faut noter que les articles qui donnent le détail de cette transaction ne sont pas retranscrits dans le journal d'annonces légales, La Gazette des tribunaux, 24 avril 1873, p. 4.

83. Ibidem, art. 5.

84. Ibid., art. 36 à 40.

(c) Gabriel Galvez-Behar 
droit à $20 \%$ des bénéfices de la Société ${ }^{85}$. Par ailleurs, en contrepartie de son concours, il lui serait versé un traitement de 25000 francs annuel sur l'excédent brut d'exploitation. Si ce dernier n'était pas assez élevé pour le lui verser, la Société s'engageait à prendre en charge le salaire des chimistes-adjoints à hauteur de 8000 francs. Enfin, en cas de liquidation anticipée de la Société, Pasteur pourrait retrouver ses droits sur son brevet sans être contraint à quelque remboursement que ce soit. Bien entendu, cette transaction reposait sur la valeur des apports de Pasteur dont l'expertise fut confiée à deux actionnaires, Antoine Schnapper et Jules Reiset, chimiste et membre correspondant de l'Académie des sciences. Lors de la seconde assemblée générale du 27 mars 1873, leur rapport conclut à l'adoption des statuts, c'est-à-dire à l'acceptation des apports de Pasteur et à « l'allocation des avantages particuliers stipulés à son profit $»^{86}$.

À la lumière de cet événement, les communications de Pasteur sur la bière dans le courant de l'année 1873 prennent une autre tonalité : elles apparaissent comme une forme de réclame pour le procédé Pasteur en cours de commercialisation tandis que les brevets pris en France et à l'étranger après le 4 mars 1873 sont autant de preuves de l'activité internationale de la société ${ }^{87}$. Cette dernière, cependant, ne semble pas avoir eu une longue activité, encore que l'on n'ait pas trouvé trace de sa dissolution. Ainsi ne sait-on pas si la récompense obtenue en 1874 vint s'ajouter aux revenus procurés par les Bières inaltérables ou si elle vint s'y 85. Ibid., art. 6. En fait, Pasteur reçut quarante actions de fondateur mais déclara en distribuer la moitié à d'autres actionnaires.

86. AN, MC/ET/LIX/709, procès-verbal de l'assemblée générale des actionnaires de La Société des bières inaltérables, 27 mars 1873.

87. Au moins un ingénieur, Marcel Magnuski, fut également embauché comme l'atteste le Bulletin de la papeterie, août 1890, p. 146 ; Marie-Cécile Bouju, «L'École Estienne, 1889-1949 » thèse pour le diplôme d'archiviste-paléographe, École des Chartes, 1998, p. 112.

(C) Gabriel Galvez-Behar Version de pré-publication/Pre-print version $40 / 53$ 
substituer après une liquidation prématurée de la société ${ }^{88}$. Toujours est-il que les 150000 francs de la cession du brevet demeuraient acquis à Pasteur qui trouvait en outre le moyen d'engager des collaborateurs pour continuer des travaux sur la bière. Le projet de devenir riche se concrétisait pour le plus grand bien de l'entreprise pasteurienne.

\section{De la Société des bières inaltérables à l'Institut Pasteur}

Tout comme l'analyse fine de la correspondance de Pasteur remet en question son prétendu désintérêt pour l'argent, tout comme celle de ses brevets nuance largement sa prétendue volonté d'enrichir le seul domaine public, l'analyse précise de la création de la Société des bières inaltérables ébranle largement le stéréotype selon lequel Pasteur se serait refusé à commercialiser ses découvertes ${ }^{89}$. D’ailleurs, comme le fait remarquer Claire Salomon-Bayet, une telle entreprise suscita l'étonnement voire la critique ${ }^{90}$. Aussi offre-t-elle une nouvelle perspective sur l'activité économique de Louis Pasteur et sur le rôle que ce dernier put jouer dans certaines sociétés.

À la mort de Dumas, Pasteur remplaça ce dernier au conseil d'administration du Crédit foncier. Quand on sait maintenant le rôle que jouèrent certains de ses principaux dirigeants dans la création de la Société des bières inaltérables, on est en droit de penser que cette nomination n'avait pas qu'une fonction honorifique. Le Crédit foncier fut ainsi l'une des

88. En 1887, Lutaud affirmait ainsi que la société n'avait pas duré bien longtemps : A. Lutaud, M. Pasteur et la rage, op. cit., p. 429.

89. Adrien Loir, « A l'ombre de Pasteur. Pasteur et l'exploitation commerciale des découvertes scientifiques », Le Mouvement sanitaire, 1937, vol. 14, p. 188-192.

90. Le 8 avril 1873, la Semaine politique, dans un article intitulé La bière de la revanche, le rédacteur dénonce «ce concubinage de la spéculation et du patriotisme » in C. Salomon-Bayet, Pasteur et la révolution pastorienne, op. cit., p. 42.

(C) Gabriel Galvez-Behar Version de pré-publication/Pre-print version 
institutions amenées à récolter des fonds pour la création de l'Institut Pasteur en 1886. Aussi faut-il se pencher un peu plus en avant sur cette dernière, permise par le succès des recherches de Pasteur sur la rage. Créé le 21 mai 1886, l'Institut Pasteur prit d'abord la forme d'une société anonyme avant que le Conseil d'État n'impose un changement des statuts compatible avec la reconnaissance d'utilité publique. Dans la biographie qu'il consacre à Pasteur, Maurice Vallery-Radot affirme que ce choix initial découlait d'un mauvais conseil ${ }^{91}$. Un tel jugement tend cependant à oublier plusieurs faits. Non seulement Pasteur n'était plus tout à fait ignorant de tels montages financiers mais encore la création de l'Institut intervint au milieu de celles de deux autres sociétés : la Compagnie de vulgarisation des vaccins charbonneux Pasteur, d'une part, la société anonyme du filtre Chamberland (système Pasteur), de l'autre. Sans entrer dans le détail de ces montages qui mériterait une étude complète, il est possible de dépeindre ce contexte à grands traits.

Tableau 4: Les sociétés impliquées dans l'entreprise pasteurienne en 1886

\begin{tabular}{|c|c|c|}
\hline Date & Nom de la société & Observations \\
\hline 19 avril 1886 & $\begin{array}{l}\text { [Traité entre Henri Lefebvre de } \\
\text { Sainte-Marie et Chamberland, au } \\
\text { nom de Pasteur, Roux et lui-même] }\end{array}$ & $\begin{array}{l}\text { Chamberland, Pasteur et Roux s'engagent à } \\
\text { concéder leur secret de fabrication du } \\
\text { vaccin contre le charbon pour sa } \\
\text { «vulgarisation » en dehors de la France } \\
\text { (l'Autriche, l'Argentine, le Paraguay et } \\
\text { l'Uruguay exceptés) }\end{array}$ \\
\hline 30 avril 1886 & $\begin{array}{l}\text { Constitution de la Compagnie de } \\
\text { vulgarisation du vaccin charbonneux } \\
\text { Pasteur }\end{array}$ & $\begin{array}{l}\text { Création par Henri Lefebvre de Sainte- } \\
\text { Marie d'une société anonyme pour } \\
\text { l'exploitation du monopole concédé par } \\
\text { Chamberland, Roux et Pasteur. }\end{array}$ \\
\hline 21 mai 1886 & $\begin{array}{l}\text { Constitution de la société anonyme } \\
\text { Institut Pasteur }\end{array}$ & $\begin{array}{l}\text { Création d'une société anonyme à capital } \\
\text { variable pour le traitement de la rage. }\end{array}$ \\
\hline 5 juin 1886 & Constitution de la société anonyme & Création d'une société anonyme pour \\
\hline
\end{tabular}

91. M. Vallery-Radot, Pasteur, op. cit., chap. 7.

(C) Gabriel Galvez-Behar Version de pré-publication/Pre-print version 


\begin{tabular}{|l|l|l|l|}
\hline & $\begin{array}{l}\text { du filtre Chamberland, système } \\
\text { Pasteur }\end{array}$ & $\begin{array}{l}\text { l'exploitation des brevets sur le filtre } \\
\text { Chamberland }\end{array}$ \\
\hline
\end{tabular}

La constitution de ces trois sociétés en l'espace de deux mois ne résulte pas d'un concours de circonstances. S'il est vrai que le processus de création de l'Institut Pasteur fut lancé à l'occasion d'une séance de l'Académie des sciences le 1886, la constitution des sociétés sur le vaccin charbonneux et sur le filtre Chamberland résulte plutôt d'une réorganisation de l'entreprise pasteurienne et d'une redistribution de ses bénéfices. Un premier élément corroborant cette hypothèse est le rôle central joué par Chamberland dans les trois affaires. Normalien, Chamberland avait intégré le laboratoire de Pasteur en 1875 et en était devenu directeur-adjoint trois ans plus tard. Il joua un rôle-clef dans la mise au point du vaccin charbonneux, notamment lors de la fameuse expérience de Pouilly-le-Fort de 1881. Scientifique à l'esprit inventif, il mit au point en 1884 un filtre permettant d'éliminer les microbes de l'eau pour lequel il prit plusieurs brevets. En 1886, il servit de fondé de pouvoir entre Henri de Sainte-Marie, ancien inspecteur du ministère de l'Agriculture, et le groupe formé par Pasteur, Roux et lui-même. Par contrat du 19 avril 1886, ces derniers concédaient au premier « le monopole exclusif de créer à l'étranger (la France et ses colonies exceptées) » des laboratoires pour la vulgarisation du vaccin charbonneux sous le nom de "Laboratoire du Vaccin charbonneux Pasteur" $\gg{ }^{92}$ En contrepartie, Chamberland recevrait, pour l'ensemble du groupe qu'il représentait, une partie des recettes proportionnelle au nombre de doses vendues.

92. Institut Pasteur, Musée Pasteur, doc. 10587.

(C) Gabriel Galvez-Behar Version de pré-publication/Pre-print version 
Chamberland ne semble pas avoir eu une implication directe dans la première création de l'Institut Pasteur en 1886. Il fut en revanche la figure centrale de la création de la Société du filtre Chamberland système Pasteur créée le 5 juin $1886^{93}$. Cette dernière visait à exploiter la douzaine de brevets pris dans le monde entier pour protéger son invention mise au point dans le laboratoire de Pasteur. Si Pasteur ne joua pas un rôle direct dans la constitution de cette société, cette dernière n'en porta pas moins son nom grâce à une autorisation que Pasteur dut donner à son collaborateur. Marqué par l'exemple de Liebig dont le nom avait fini par se retrouver sur des produits alimentaires, Pasteur était, en effet, extrêmement soucieux de contrôler l'usage de son nom. Une modification des statuts de la société précisa d'ailleurs que parmi les apports en nature les marques de fabrique devaient être prises en compte. Le nom de Pasteur devenait ainsi un apport en nature de la société du filtre Chamberland.

Il faut toutefois attendre la nouvelle création de l'Institut Pasteur en 1887 pour bien mesurer le lien entre ces différentes sociétés. L'année 1886 avait été mise à profit pour recueillir des fonds dans le cadre d'une souscription centralisée par le Crédit foncier qui avait proposé ses bons offices dès 1886. Christophle, sous-gouverneur du Crédit foncier, figurait ainsi parmi les premiers actionnaires de l'Institut Pasteur. Le poids de la banque parisienne fut d'ailleurs essentiel dans la réussite de la souscription puisque près de 200000 francs provinrent d'établissements financiers, dont 40000 francs pour le Crédit foncier et 40000 pour la banque Rothschild ${ }^{94}$. Toutefois, pour reconnaître l'utilité publique de l'Institut, le Conseil d'État fit valoir qu'il était nécessaire d'en modifier les statuts. Le caractère de société 93. AN, MC/ET/LXXXVII/1916, Statuts de la Société du Filtre Chamberland. 94. Institut Pasteur, Service des archives, DR FND 1, doc. 18428.

(C) Gabriel Galvez-Behar Version de pré-publication/Pre-print version 
anonyme à capital variable n'était guère jugé approprié pour un établissement de la sorte. Une réécriture des statuts fut ainsi entreprise à l'hiver 1887 et Chamberland tout comme Duclaux furent largement consultés par Pasteur. À l'occasion d'une Assemblée générale de l'Institut réunie le 21 janvier 1887, Jules Simon réclama, «pour répondre à un scrupule qu'il [avait] entendu exprimer, qu'un paragraphe additionnel soit inséré, spécifiant expressément que les membres de la Société de l'Institut Pasteur ne peuvent trouver, ni bénéfice, ni rémunération pécuniaire d'aucune sorte ${ }^{95}$. Jules Simon posait implicitement la question du désintéressement de l'entreprise pasteurienne.

Dans une lettre adressée au président du Comité, Pasteur indiqua qu'après la mise au point du vaccin contre le charbon il avait été nécessaire d'installer à la hâte une officine de préparation et un bureau d'expédition. Domicilié rue Vauquelin, grâce à une subvention du ministère de l'Agriculture, ce laboratoire, portant la raison sociale du beau-frère de son fils, Fernand Boutroux, fut chargé de vendre des doses de vaccins aux agriculteurs français. Pasteur rappela la proposition qu'il avait faite, dès l'automne 1881, à Gambetta puis à Méline, d'offrir à l'État le service spécial de la livraison du vaccin charbonneux pour la France ${ }^{96}$. Il suggérait dorénavant au président du Comité de l'Institut Pasteur de rattacher à ce dernier les services de fabrication des vaccins contre le charbon, le choléra des poules et le rouget des porcs. Les dépenses et les recettes de ce service seraient dès lors versées au budget de l'Institut. Le 25 mars 1887, lors d'une séance du Comité tenue en l'absence de Pasteur, le professeur Grancher, ami et allié de ce dernier à l'Académie de médecine, présenta le contenu 95. Institut Pasteur, Service des archives, CAD REG 1, procès-verbaux du Comité, 21 janvier 1887. 96. Louis Pasteur, Correspondance de Pasteur, 1840-1895, Paris, Flammarion, 1951, vol. 4, p. 173-174.

(C) Gabriel Galvez-Behar Version de pré-publication/Pre-print version 
de cette lettre en déclarant que Pasteur abandonnait « le bénéfice de la vente du vaccin charbonneux, de celui du rouget et du choléra des poules. ${ }^{97}$ Jules Simon remercia alors Pasteur de sa générosité, ajoutant que « cet important revenu [était] la propriété légitime de M. Pasteur et de ses collaborateurs. ${ }^{98}$

En donnant à comprendre qu'il apportait à l'Institut le fruit de ses découvertes, Pasteur parvenait à obtenir la reconnaissance $a$ posteriori de sa « propriété ». Pourtant, la présentation faite par Grancher n'était rien moins que partielle. La disposition statutaire qui consacrait cet apport était beaucoup moins large que ce qu'il avait bien voulu faire croire. L'alinéa 4 de l'article 22 des nouveaux statuts de l'Institut adoptés le 20 mai 1887, prévoyait ainsi que « les bénéfices de la livraison en France des vaccins découverts dans le laboratoire de M. Pasteur, rue d'Ulm 45, avant la fondation de l'Institut » seraient compris dans les recettes annuelles. Une telle rédaction excluait non seulement les découvertes à venir mais, surtout, le bénéfice des vaccins livrés à l'étranger. Ainsi étaient préservés les monopoles concédés pour l'Autriche, l'Argentine, l'Uruguay et le Paraguay mais aussi pour le reste du monde par l'intermédiaire de la Compagnie de vulgarisation du vaccin charbonneux Pasteur. Enfin, dans l'esprit de Pasteur, les bénéfices concédés à l'Institut ne pouvaient être que les siens propres. Dans une note en marge des nouveaux statuts, Pasteur précisa, en effet, que l'abandon des bénéfices de Chamberland et de Roux devait être compensé par une augmentation de traitement $^{99}$. Ainsi, malgré l'annonce faite au Comité, Pasteur n'avait pas tout à fait renoncé à

97. Institut Pasteur, Service des archives, CAD REG 1, procès-verbaux du Comité, 25 mars 1887.

98. Idem.

99. Institut Pasteur, doc. 18 450, Institut Pasteur, statuts du 20 mai 1887, exemplaire avec notes en marge.

(C) Gabriel Galvez-Behar Version de pré-publication/Pre-print version 
des bénéfices personnels, pour lui et ses collaborateurs, sur les vaccins destinés à un usage vétérinaire.

Ce partage ne se fit sans doute pas sans heurts. Quelques temps après l'adoption des nouveaux statuts de l'Institut, Roux écrivit à Chamberland en ces termes :

«Mon cher Chamberland,

L'année dernière je vous ai donné, en ce qui me concerne, tout pouvoir pour traiter de l'exploitation du vaccin charbonneux à l'étranger. Vous vous êtes entendu, pour cette exploitation, avec M. de Sainte-Marie qui, d'après ce que j'ai appris, a formé une société dans ce but. Je désire rester étranger à des combinaisons financières que je ne puis suivre. Je viens donc vous dire que je renonce à toute participation à cette affaire et que je remets en vos mains la somme de 10000 francs que vous m'avez donnée comme représentant ma part. » ${ }^{100}$

Roux dut cependant se laisser convaincre de renoncer à sa renonciation. En effet, un document de Chamberland permet de se faire une idée du partage des profits engendrés par la commercialisation des vaccins à l'étranger ${ }^{101}$. Intitulé «Compte vaccins étrangers », ce petit cahier de quatre pages permet d'évaluer les redevances touchées par Pasteur, Roux et Chamberland. Ainsi, pour les années 1889,1890 et 1891, la vente de vaccins en AutricheHongrie rapporta-t-elle aux trois savants 17740,26801 et 25000 francs. La vente des vaccins en Inde au premier trimestre 1891 leur rapporta 10000 francs. Pour l'Australie, Sainte-Marie remit, le 8 juin 1891, 5751 francs à Chamberland qui, dès le lendemain, donna leurs parts à Pasteur et à Roux. L'étude de ce document montre que la part de Pasteur représentait le double de celle de ses deux associés. Ainsi, pour l'année 1891, Pasteur avait-il reçu près de 21000 francs, ce qui correspondait presque à sa pension obtenue après 100. Institut Pasteur, service des archives, DR DOS 1, lettre de Roux à Chamberland, 28 mai 1887. 101. Institut Pasteur, doc. 10 610, comptes vaccins étrangers.

(c) Gabriel Galvez-Behar Version de pré-publication/Pre-print version 
l'augmentation de sa récompense nationale ${ }^{102}$. Sa générosité et celle de ses plus proches collaborateurs s'étaient ainsi limitées aux bénéfices tirés de l'exploitation des vaccins vétérinaires en France et sans doute du vaccin contre la rage. Cette contribution n'était pas mince mais, pour le reste, Pasteur et les siens avaient fait valoir leur « propriété légitime ».

\section{$\underline{\text { La fortune de Louis Pasteur }}$}

Ainsi, tout au long de sa carrière, Pasteur ne négligea ni ses intérêts personnels ni ceux de ses plus proches collaborateurs engagés dans l'entreprise pasteurienne. Si le succès scientifique ou symbolique de cette dernière ne fait aucun doute, reste à en mesurer le résultat sur un plan tout à fait matériel. À cet égard, les opérations de succession entreprises après le décès de Pasteur en septembre 1895 permettent de se faire une idée précise de sa fortune ${ }^{103}$.

Tableau 5: Tableau simplifié de l'actif de succession de Louis Pasteur (AN, MI, RS, 1173)

\begin{tabular}{|l|r|}
\hline Désignation de l'actif & Montant (en francs) \\
\hline Mobilier et objets personnels & 15000,00 \\
\hline Liquidités et comptes courants & 9685,65 \\
\hline Prorata d'arrérages des pensions civiles de M. Pasteur & 10161,10 \\
\hline Assurance-vie de M. Pasteur & 35056,55 \\
\hline Actions et obligations du Crédit foncier de France & 173794,85 \\
\hline Obligations communales & 89702,50 \\
\hline Obligations chemins de fer d'Orléans et Paris-Lyon-Marseille & 205940,50 \\
\hline Autres obligations & 5345,25 \\
\hline Rente russe & 56115,00 \\
\hline Actions & 7600,00 \\
\hline
\end{tabular}

102. Ce document comporte même l'indication suivante : « Le 23 mars 1892 j'ai remis à M. Pasteur son compte de filtre : 6000 francs pour Autriche-Hongrie, à M. Roux 3000 francs pour Autriche-Hongrie ». Cette mention du filtre laisse penser que Pasteur et Roux touchaient également des redevances pour l'exploitation du filtre Chamberland.

103. AN, MI/RS/1173, [MC/ET/XLIII/1500], liquidation de la succession de Louis Pasteur, 13 mars 1896. Le passif de succession est quasiment nul.

(c) Gabriel Galvez-Behar Version de pré-publication/Pre-print version 


\begin{tabular}{|l|r|}
\hline Maisons et vignes à Arbois et à Montigny (Jura) & 20000,00 \\
\hline Divers & 651,50 \\
\hline Rapport d'avances et de dot & 400000,00 \\
\hline Total & 1029052,90 \\
\hline
\end{tabular}

S'il n'est pas tout à fait exceptionnel dans le milieu universitaire, ce niveau de fortune place Pasteur dans le quartile le plus riche voire dans les $15 \%$ des universitaires les plus fortunés ${ }^{104}$. La fortune de Pasteur le détache nettement de la grande majorité de ses collègues pour le rapprocher des professeurs de médecine, des inspecteurs des mines, des administrateurs de grandes sociétés. Sa fortune n'atteint pas, en revanche, le niveau des grands brasseurs d'affaires dont la fortune médiane atteint à la fin du $\mathrm{XIX}^{\mathrm{e}}$ siècle plus de 3 millions de francs ${ }^{105}$.

L'enrichissement de Pasteur est autrement plus singulier. Comparé à sa fortune initiale ou à celle de sa femme, le taux d'enrichissement varie entre 1450 et $9300 \%$. Ces chiffres, sans commune mesure avec les autres taux connus au sein des élites françaises de la fin du XIX siècle, traduisent le caractère exceptionnel de la trajectoire de Pasteur non seulement sur le plan scientifique mais aussi sur le plan social et financier ${ }^{106}$. La composition de cette fortune s'avère également exceptionnelle avec la nette sur-représentation des obligations dont le montant dépasse $60 \%$ du patrimoine total ${ }^{107}$. Comparée aux autres fortunes de la même époque, celle de Pasteur reflète une forte aversion au risque - comme si tout avait été fait pour garantir ce spectaculaire enrichissement de tout revers de fortune. À la fin de sa vie, Pasteur

104. Christophe Charle, Les élites de la république : 1881-1900, Paris, France, Fayard, 1987, p. 359.

105. Ibid., p. 365.

106. Ibid.

107. Le calcul est rapporté au montant total de l'actif soustraction faite des dots et avances.

(C) Gabriel Galvez-Behar Version de pré-publication/Pre-print version 
voyait ainsi exaucé le vœu qu'il avait formulé une trentaine d'année plus tôt : celui de mettre sa famille à l'abri du besoin.

\section{CONCLUSION}

Terminons donc de rassembler tous les faits car ils sont au cœur de la question. En dépit de la longue tradition hagiographique, il faut bien reconnaître que Pasteur développa tout au long de sa carrière un intérêt certain pour l'argent et qu'il eut un usage longtemps ambigu des brevets d'invention avant de se décider à en tirer profit grâce à de grandes banques parisiennes. Impliqué dans des montages financiers avec certains de ses collaborateurs, il recueillit les bénéfices de l'exploitation commerciale à l'étranger de ses vaccins vétérinaires alors que l'Institut Pasteur, financé en partie grâce aux mêmes banques qui avaient déjà aidé son fondateur, restait cantonné dans une logique apparemment désintéressée. Ce double jeu permit à Pasteur d'accumuler une fortune conséquente, fruit d'un enrichissement exceptionnel pour un universitaire d'origine modeste.

Ces faits suffiraient à casser de manière définitive l'image du Pasteur désintéressé mais tel n'est pas leur but. Ils permettent plutôt de montrer les facteurs économiques et même financiers au cœur de l'entreprise scientifique pasteurienne. Décrite comme un processus de déplacement, cette dernière se ramène, selon Bruno Latour, à deux principes : Pasteur se déplace vers des problèmes toujours moins fondamentaux mais impliquant toujours plus de monde tandis qu'il transforme les problèmes appliqués en problèmes fondamentaux résolus grâce aux moyens acquis dans la discipline qu'il vient de quitter ${ }^{108}$. Or notre histoire 108. B. Latour, Pasteur..., op. cit., p. 113. 
économique de l'entreprise pasteurienne montre bien que ces moyens ne sont pas seulement épistémiques ou techniques. Ils ne sont pas seulement des microbes, des microscopes, des communications. Ce sont aussi de l'argent, des brevets voire du capital.

Ces points d'appui ne sont pas distribués de manière aléatoire. Il est tout à fait symptomatique de remarquer, en effet, la place récurrente des sollicitations financières ou des demandes de brevets. Ces dernières, par exemple, sont faites à des moments bien particuliers des déplacements pasteuriens, notamment quand Pasteur s'engage dans un champ nouveau, exception faite des travaux sur le ver à soie ou sur les vaccins. Ce qui fait le succès des déplacements pasteuriens, c'est qu'ils sont intégrés à une démarche économique qui lie le projet entrepreneurial aux ressources nécessaires à sa mise en œuvre. Cette dimension économique ne se limite cependant pas à un rapport des moyens aux fins. Elle possède aussi une portée axiologique destinée à mettre en avant la valeur voire les droits de la science. Non seulement l'entreprise pasteurienne repose sur des besoins matériels clairement explicités mais encore elle permet des bénéfices dont les acteurs, à commencer par Pasteur, ont clairement conscience. Cette question de la distribution de la valeur ajoutée pasteurienne, Pasteur et les siens ne la négligent pas. Aussi n'hésitent-ils pas à faire valoir leurs droits au profit quitte à écorner le dogme de la gratuité de la science. Pour eux, la science a non seulement un coût mais elle a un prix.

Ainsi considérée, l'entreprise pasteurienne pourrait être comprise à bon droit comme un processus d'accumulation. L'enrichissement personnel de Pasteur permettrait d'ailleurs de donner du sens à une telle affirmation. Encore faut-il ne pas oublier que ces gains furent 
réinvestis dans le champ scientifique pour mener des projets toujours un peu plus risqués. Si le chauffage du vin peut gâter partiellement le fruit d'une récolte, une campagne de vaccination contre le charbon peut tuer un cheptel. Le risque est plus grand encore quand il s'agit de vacciner des êtres humains. Aussi le processus d'accumulation financière peut-il être interprété comme l'envers d'une entreprise toujours plus risquée sur le plan scientifique. La gestion très prudente de la fortune personnelle de Pasteur apparaîtrait comme l'envers de l'entreprise scientifique parfois périlleuse. C'est en cela, aussi, que Pasteur s'avère être pleinement un entrepreneur scientifique.

Reste à comprendre, enfin, pourquoi cette dimension économique si essentielle a pu être sous-estimée voire refoulée. Élément essentiel de l'entreprise pasteurienne, ce mécanisme de dénégation est suffisamment connu pour ne relever ici que son aspect économique. La construction du mythe pasteurien est une partie intégrante de l'entreprise car la mise en exergue du désintéressement est précisément ce qui permet d'attirer des capitaux nouveaux. C'est la raison pour laquelle cette quasi-vertu cardinale est mise en avant quand sont négociées les récompenses ou les souscriptions. C'est aussi la raison pour laquelle elle est mise en doute par les adversaires de Pasteur à des moments aussi stratégiques.

Une autre facette de cette dénégation tient aux traces que l'entreprise pasteurienne a laissées. Il est tout à fait significatif, en effet, que les correspondances commerciales aient quasiment disparu de l'importante masse de sources laissées par Pasteur et par les siens. Sans vouloir faire planer un soupçon stérile, tout porte à croire maintenant que les acteurs de l'entreprise pasteurienne n'ont laissé derrière eux que les traces qui correspondaient à (c) Gabriel Galvez-Behar Version de pré-publication/Pre-print version 
l'histoire qu'ils voulaient voir écrite. Et c'est aussi sur ce terrain qu'une histoire économique des mondes savants doit se placer. Car l'histoire économique n'est pas tant une subdiscpline définie par un objet ou par une méthodologie bien arrêtés qu'une tradition heuristique qui mène l'enquête là où d'autres ne vont pas.

Gabriel GALVEZ-BEHAR

Univ. Lille, CNRS, UMR 8529 - IRHiS Institut de Recherches Historiques du Septentrion, F-59000 Lille, France

Institut universitaire de France 